\title{
Lung vaso-occlusion in sickle cell disease mediated by arteriolar neutrophil-platelet microemboli
}

\author{
Margaret F. Bennewitz, ${ }^{1}$ Maritza A. Jimenez, ${ }^{1,2}$ Ravi Vats, ${ }^{1,2}$ Egemen Tutuncuoglu, ${ }^{1}$ Jude Jonassaint, ${ }^{1,3}$ \\ Gregory J. Kato, ${ }^{1,3}$ Mark T. Gladwin, ${ }^{1,4}$ and Prithu Sundd ${ }^{1,2,4}$ \\ 'Pittsburgh Heart, Lung and Blood Vascular Medicine Institute, University of Pittsburgh School of Medicine, Pittsburgh, \\ Pennsylvania, USA. ${ }^{2}$ Department of Bioengineering, University of Pittsburgh, Pittsburgh, Pennsylvania, USA. \\ ${ }^{3}$ Division of Hematology and Oncology, and ${ }^{4}$ Division of Pulmonary Allergy and Critical Care Medicine, \\ University of Pittsburgh School of Medicine, Pittsburgh, Pennsylvania, USA.
}

In patients with sickle cell disease (SCD), the polymerization of intraerythrocytic hemoglobin S promotes downstream vaso-occlusive events in the microvasculature. While vaso-occlusion is known to occur in the lung, often in the context of systemic vaso-occlusive crisis and the acute chest syndrome, the pathophysiological mechanisms that incite lung injury are unknown. We used intravital microscopy of the lung in transgenic humanized SCD mice to monitor acute vaso-occlusive events following an acute dose of systemic lipopolysaccharide sufficient to trigger events in SCD but not control mice. We observed cellular microembolism of precapillary pulmonary arteriolar bottlenecks by neutrophil-platelet aggregates. Blood from SCD patients was next studied under flow in an in vitro microfluidic system. Similar to the pulmonary circulation, circulating platelets nucleated around arrested neutrophils, translating to a greater number and duration of neutrophilplatelet interactions compared with normal human blood. Inhibition of platelet P-selectin with function-blocking antibody attenuated the neutrophil-platelet interactions in SCD patient blood in vitro and resolved pulmonary arteriole microembolism in SCD mice in vivo. These results establish the relevance of neutrophil-platelet aggregate formation in lung arterioles in promoting lung vasoocclusion in SCD and highlight the therapeutic potential of targeting platelet adhesion molecules to prevent acute chest syndrome.

Authorship note: M.F. Bennewitz and M.A. Jimenez contributed equally to this manuscript and are co-first authors.

Conflict of interest: The authors have declared that no conflict of interest exists.

Submitted: July 27, 2016 Accepted: November 29, 2016 Published: January 12, 2017

Reference information: JCI Insight. 2017;2(1):e89761. doi:10.1172/jci.insight.89761.

\section{Introduction}

Sickle cell disease (SCD) is one of the most common monogenetic disorders in the world (1). SCD affects over 3 million people worldwide and results in at least 100,000 deaths every year (2). Sickle cell anemia, the most common form of SCD, is caused by a homozygous mutation (SS) in the $\beta$-globin gene. The mutant hemoglobin $(\mathrm{HbS})$ polymerizes when deoxygenated, forming intraerythrocytic polymer bundles that lead to cellular rigidity, dehydration, impaired rheology, and premature hemolysis (3). Two dominant pathophysiological events have been characterized: the intravascular entrapment of erythrocytes and inflammatory cells leading to occlusion of the microvasculature (vaso-occlusion); and intravascular hemolysis, which produces anemia and endothelial injury and dysfunction (3). Chronic hemolysis may also prime leukocytes, platelets, and the vascular endothelium to promote vaso-occlusion in response to an inflammatory or environmental stimulus (4). Acute vaso-occlusive painful crisis is the predominant pathophysiology requiring emergency medical care by SCD patients (3). Repeated episodes of vaso-occlusive crisis contribute to morbidity and end organ damage in SCD patients, and there is a strong need for improved preventive strategies $(1,3)$. Acute vaso-occlusive crisis can involve the lung, bone marrow, and systemic blood vessels $(3,5)$. Systemic vaso-occlusive events have been characterized in transgenic mouse models of SCD and in the human retinal vasculature, and appear to be mediated by vaso-occlusive events in the postcapillary venules. While the lung is one of the most affected organs in SCD (5), the cellular, molecular, anatomic, and biophysical mechanisms that contribute to pulmonary vaso-occlusion have not been characterized (3).

The humanized transgenic Berkeley (BERK) SCD mouse, which almost exclusively expresses human sickle hemoglobin (6), has been shown to mimic human SCD pathophysiology, such as sickling and hemo- 
lysis of erythrocytes, increased hematopoiesis, intravascular congestion, and multi-organ infarction (7). Intravital microscopic studies of the cremaster microcirculation of transgenic SCD mice have shown that systemic vaso-occlusion occurs primarily in the postcapillary venules, involving CD11b/CD18 (Mac-1)dependent capture of sickle erythrocytes by adherent neutrophils (8). Recently, intravital microscopy of the TNF- $\alpha$-treated cremaster venules in WT mice revealed that platelets can interact through P-selectin binding to P-selectin glycoprotein ligand-1 (PSGL-1) on adherent neutrophils (9). Additionally, neutrophil-platelet aggregates were observed in TNF- $\alpha$-treated cremaster venules of SCD mice, requiring neutrophil Mac- 1 binding to glycoprotein $\mathrm{Ib} \alpha(\mathrm{GPIb} \alpha)$ on platelets (10). Interestingly, elevated platelet and leukocyte counts are identified risk factors for acute vaso-occlusive painful crisis (11), and circulating neutrophil-platelet aggregates are significantly elevated in the blood of steady-state SCD patients $(12,13)$. Recent studies also suggest that neutrophil-platelet aggregates contribute to pulmonary inflammation in WT mice in the setting of acute lung injury $(9,14)$. Despite this recent appreciation of a role of neutrophil-platelet aggregates in systemic vaso-occlusion and acute lung injury, the role of these cellular aggregates in pulmonary vaso-occlusion associated with SCD is not known. These questions can be addressed using advanced intravital microscopic investigation of the lung in SCD mice to elucidate whether and how neutrophil-platelet aggregates can promote pulmonary vaso-occlusion in SCD.

Until recently (15), intravital microscopy of the live mouse lung has been challenging due to the motion artifacts caused by breathing and beating of the heart. To address these challenges, we introduced quantitative fluorescence intravital lung microscopy (qFILM) and used it to establish that pulmonary vaso-occlusion is absent in transgenic SCD mice under baseline conditions (16). A recent finding suggests that gut microbiota-derived Toll-like receptor ligands can promote vaso-occlusion in SCD (17). Here, we use qFILM to reveal that pulmonary vaso-occlusion occurs selectively in SCD mice following an intravenous (IV) challenge with a low-nanogram dose of LPS. This finding is consistent with the clinical appreciation that mild-to-moderate inflammatory or infectious stimuli, insufficient to produce acute lung injury in normal individuals, may trigger lung injury in patients with SCD (18). Surprisingly, we found that unlike systemic vaso-occlusion, which never occurs in arterioles (1), pulmonary vaso-occlusion involved microembolism of precapillary pulmonary arterioles by large neutrophil-platelet aggregates in the lungs of SCD mice. Using our recently developed in vitro quantitative microfluidic fluorescence microscopy (qMFM) approach (19), we also observed neutrophil-platelet aggregate formation in blood from SCD patients flowing through silicone micro-channels at physiological shear stress. Finally, we provide proof of principle that specifically targeting platelet P-selectin decreases neutrophil-platelet aggregate formation in SCD patient blood in vitro and attenuates large neutrophil-platelet aggregates to resolve pulmonary arteriole microembolism in SCD mice in vivo.

\section{Results}

A low-nanogram dose of LPS triggers pulmonary vaso-occlusion selectively in SCD mice. Subtle inflammatory or environmental stimuli that are innocuous to healthy humans are observed to trigger acute vaso-occlusive pain crisis and acute chest syndrome in SCD patients $(3,5,18,20-22)$. Recently, the translocation of Toll-like receptor-4 (TLR-4) ligands from the gut microbiota into the blood circulation was found to promote systemic vaso-occlusion in SCD but not control mice (17). Bacterial LPS has also been shown to promote vaso-occlusion in skin venules of SCD mice (23). Based on these findings, we hypothesized that an intravascular challenge with a low dose of the TLR-4 ligand LPS may selectively induce pulmonary vaso-occlusion in SCD but not control mice. qFILM was used to monitor early vaso-occlusive events in the intact lung of live SCD and control mice challenged with low doses of IV LPS or physiological saline (see Methods for details). Pulmonary vaso-occlusion was rare but still more frequent in SCD than control mice administered IV saline. Remarkably, a very low dose of $0.1 \mu \mathrm{g} / \mathrm{kg}$ IV LPS (2.5 ng LPS administered to a 25 -g mouse) readily triggered pulmonary vaso-occlusion in SCD mice (Figure $1 \mathrm{~A})$, with little effect in control mice (Figure 1B).

Neutrophil-platelet aggregates sequester in arteriolar bottlenecks to enable vaso-occlusion. qFILM revealed that 0.1 $\mu \mathrm{g} / \mathrm{kg}$ IV LPS-triggered pulmonary vaso-occlusions were mediated by large neutrophil-platelet aggregates, which were unable to pass through the "arteriolar bottlenecks" located at the junction of pulmonary arterioles and capillaries. In contrast, only rare pulmonary vaso-occlusions were observed in control mice (Supplemental Figure 1A and Supplemental Video 1; supplemental material available online with this article; https://doi.org/10.1172/jci.insight.89761DS1) and SCD mice (Supplemental Figure 1B and Supplemental 

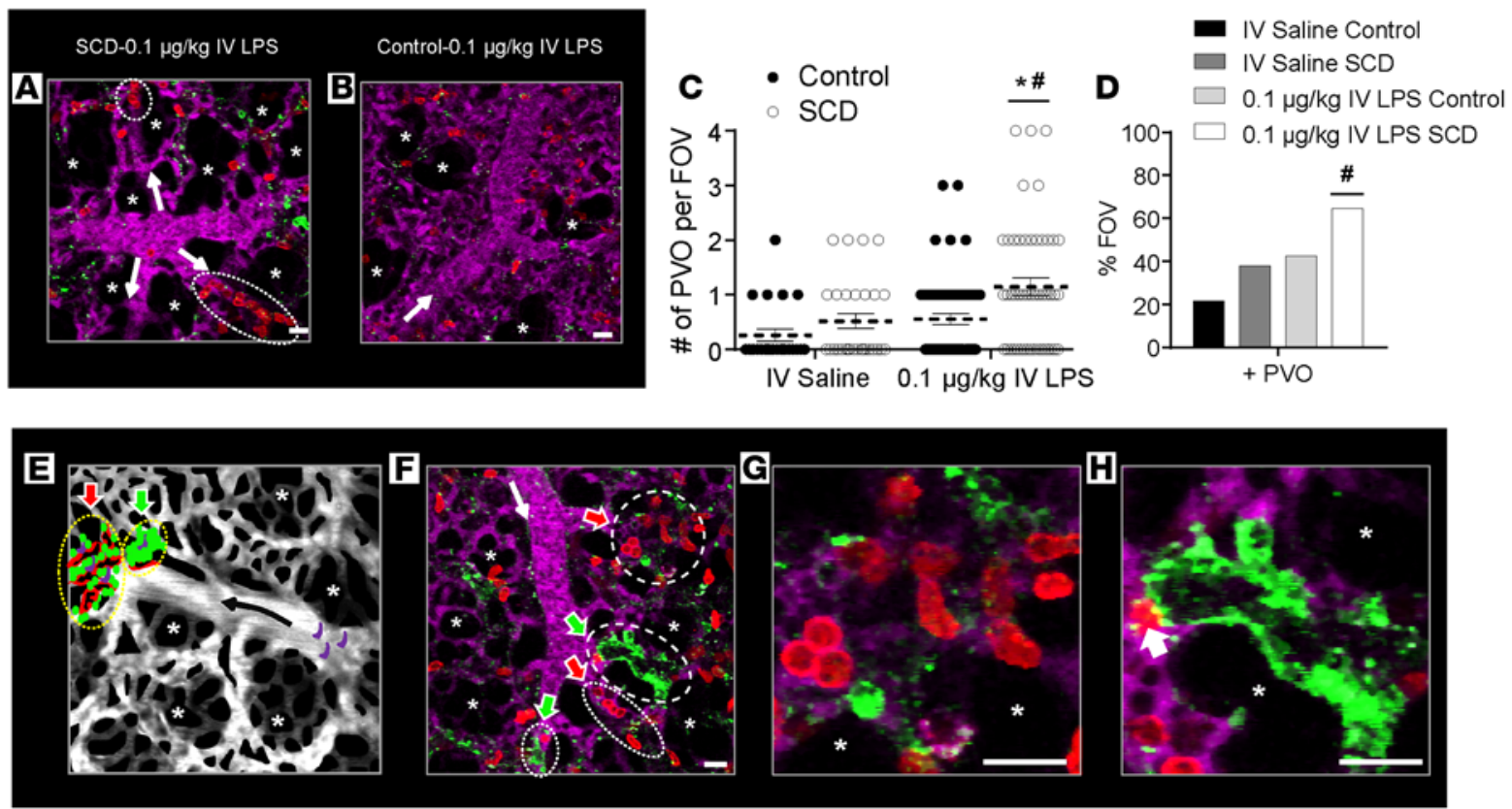

I - IV Saline Control - IV Saline SCD
$\Delta 0.1 \mu \mathrm{g} / \mathrm{kg}$ IV LPS Control $\triangle 0.1 \mu \mathrm{g} / \mathrm{kg}$ IV LPS SCD

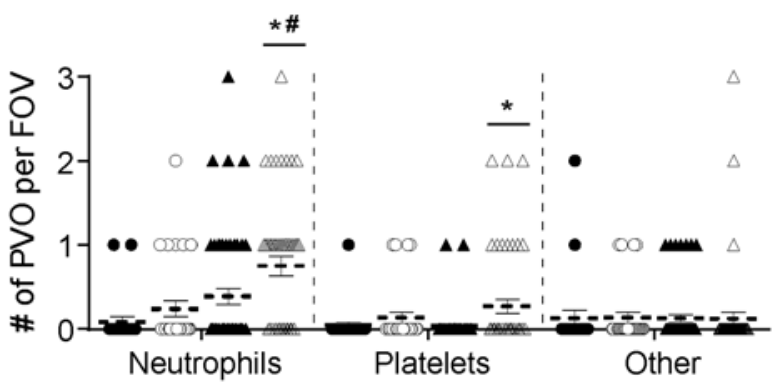

J - IV Saline Control $\Delta 0.1 \mu \mathrm{g} / \mathrm{kg}$ IV LPS Control ○ IV Saline SCD $\quad \Delta 0.1 \mu \mathrm{g} / \mathrm{kg}$ IV LPS SCD

Figure 1. $0.1 \mu \mathrm{g} / \mathrm{kg}$ IV LPS triggers pulmonary vaso-occlusion in SCD mice. Control and sickle cell disease (SCD) mice were injected intravenously (IV) with saline ( $n=3$ mice per group; control: 23 fields of view [FOVs]; SCD: 29 FOVs) or $0.1 \mu \mathrm{g} / \mathrm{kg}$ LPS ( $n=5$ mice per group; control: 54 FOVs; SCD: 48 FOVs). Arterioles were imaged $2-2.5$ hours after IV saline or $0.1 \mu \mathrm{g} / \mathrm{kg}$ IV LPS using qFILM. (A and B) qFILM images showing 2 pulmonary vaso-occlusions (dotted ellipses) in SCD mice (A) and an absence of pulmonary vaso-occlusions in control mice (B) administered $0.1 \mu \mathrm{g} / \mathrm{kg}$ IV LPS. Supplemental Video 3 shows the FOV in A. Pulmonary arteriolar vaso-occlusions were quantified as described in Supplemental Methods. (C) Number of pulmonary vaso-occlusions (PVO) per FOV for control (black circles) and SCD mice (white circles) administered IV saline or IV LPS. Size of FOV: 67,600 $\mu \mathrm{m}^{2}$. (D) Percent FOVs with pulmonary vaso-occlusions in SCD and control mice administered IV saline or IV LPS. (E) Conceptual schematic of pulmonary vaso-occlusions. Large neutrophil-platelet aggregates (yellow dotted ellipses) block arteriolar bottlenecks. Neutrophil vaso-occlusion (red arrow): few platelets (green) adhered to a neutrophil macro-aggregate (red). Platelet vaso-occlusion (green arrow): few neutrophils embedded inside a platelet macro-aggregate. Erythrocytes (purple) are trapped within neutrophil-platelet aggregates. Black arrow denotes direction of blood flow. Asterisks denote alveoli. (F) qFILM image of a SCD mouse challenged with $0.1 \mu \mathrm{g} / \mathrm{kg}$ IV LPS. All 4 arteriolar bottlenecks are blocked by neutrophil (red arrows) or platelet (green arrows) vaso-occlusions. (G) Neutrophil vaso-occlusion (magnified from F) showing mostly neutrophils (red) bound to few platelets (green). (H) Platelet vaso-occlusion (magnified from F) showing mostly platelets bound to one neutrophil (white arrow). Pulmonary microcirculation is shown in purple. White arrows in $\mathbf{A}, \mathbf{B}$, and $\mathbf{F}$ denote direction of blood flow. Diameter of arteriole shown in $\mathbf{F}$ is $\sim 28 \mu \mathrm{m}$. Scale bars: $20 \mu \mathrm{m}$. Supplemental Video 7 shows the FOV in $\mathbf{F}$. (I) Number of pulmonary vaso-occlusions per FOV classified by cellular composition. (J) Number of pulmonary vaso-occlusions per FOV with area $<1,000$ $\mu \mathrm{m}^{2}$ or $>1,000 \mu \mathrm{m}^{2}$. Average number of pulmonary vaso-occlusions per FOV, cellular composition of pulmonary vaso-occlusions, and area of pulmonary vaso-occlusions were compared using $t$ tests with Bonferroni correction. Percent of FOVs with pulmonary vaso-occlusions were compared using 4-fold table analyses with Bonferroni $\chi^{2}$ statistics. Data represent mean \pm SEM. ${ }^{*} P<0.05$ for control vs. SCD and ${ }^{*} P<0.05$ for IV saline vs. $0.1 \mu \mathrm{g} / \mathrm{kg}$ IV LPS.

Video 2) administered IV saline. Supplemental Videos 1 and 2 show freely circulating neutrophils (red) traveling up the arteriolar branches (purple) and into the capillaries (purple). Although a majority of the fields of view (FOVs) in SCD mice administered with IV saline did not show pulmonary vaso-occlusion, surprisingly, occasional aggregates comprising neutrophils and platelets were observed blocking the blood flow in arteriolar bottlenecks (Supplemental Figure 2). With $0.1 \mu \mathrm{g} / \mathrm{kg}$ IV LPS, a majority of the FOVs in SCD mice demonstrated large neutrophil-platelet aggregates trapped in the arteriolar bottlenecks (Figure 

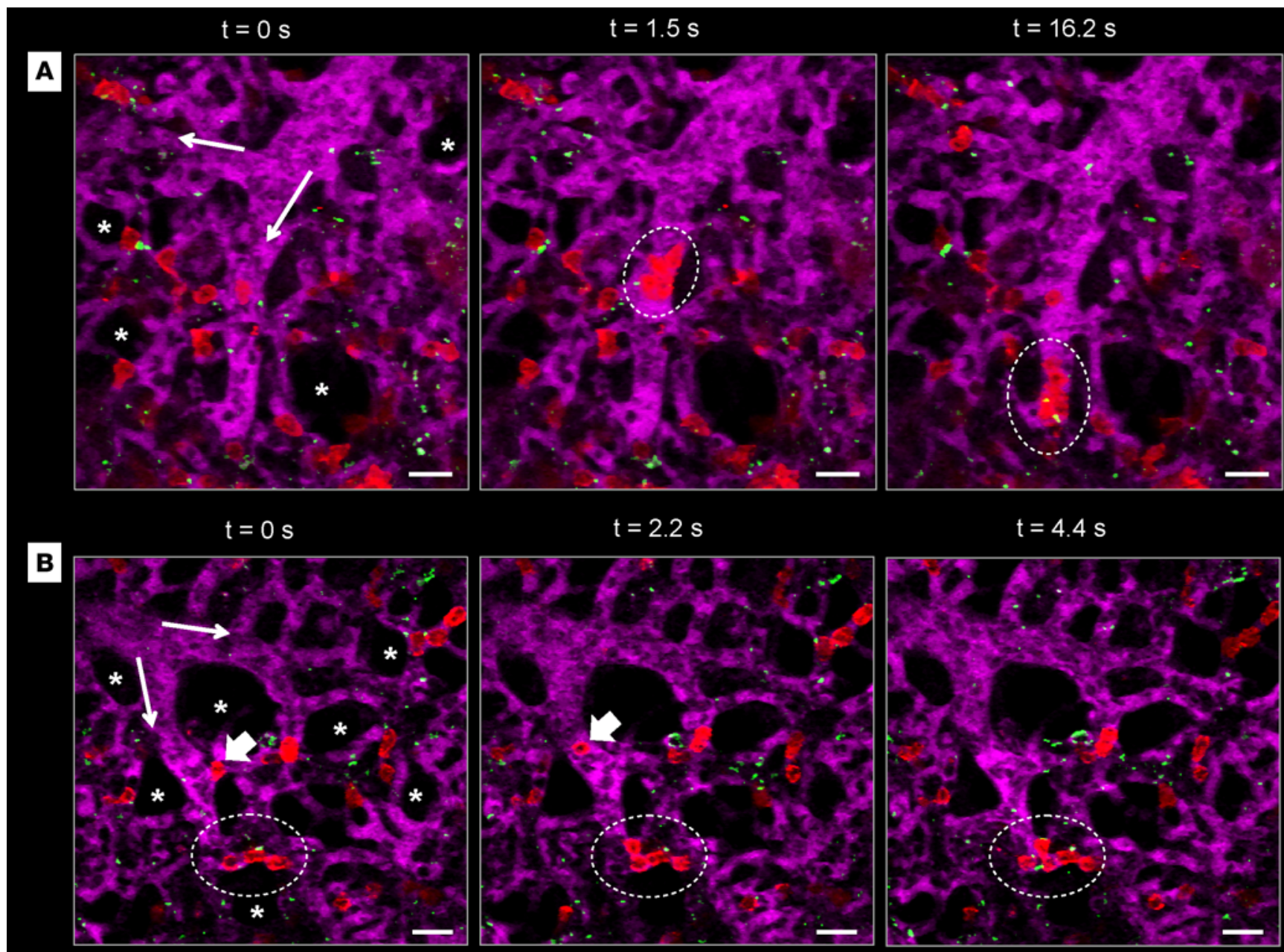

Figure 2. Microembolic or in situ nucleated neutrophil-platelet aggregates occlude the arteriolar bottlenecks. Sickle cell disease (SCD) mice were injected intravenously (IV) with $0.1 \mu \mathrm{g} / \mathrm{kg}$ of LPS, and arterioles were imaged using quantitative fluorescence intravital lung microscopy (qFILM) to evaluate the formation of pulmonary vaso-occlusion within arteriolar bottlenecks. (A) qFILM images of the same field of view (FOV) at 3 different time points in a SCD mouse administered $0.1 \mu \mathrm{g} / \mathrm{kg}$ IV LPS showing pulmonary vaso-occlusion enabled by a microembolic large neutrophil-platelet aggregate. $t=0 \mathrm{~s}$ shows the arteriole before the microembolus appears in the FOV. At $t=1.5$ seconds, a microembolus comprising several neutrophils attached to a few platelets appears (white dotted circle) and begins to travel down the arteriole. The microembolus gets trapped in the "arteriolar bottleneck," resulting in a pulmonary vaso-occlusion by $t=16.2$ seconds. (B) qFILM images of the same FOV at 3 different time points in a SCD mouse administered $0.1 \mu \mathrm{g} / \mathrm{kg}$ IV LPS showing the formation of a pulmonary vaso-occlusion via an in situ nucleation of a large neutrophil-platelet aggregate. At $t=0$ seconds, an aggregate comprising 3 neutrophils attached to a few platelets (dotted white circle) is partially occluding an arteriolar bottleneck. A neutrophil (thick white arrow) begins to travel down the arteriole at $t=0$ seconds and nucleates on top of the existing neutrophil-platelet aggregate by $t=2.2$ seconds. Another neutrophil (thick white arrow) appears in the FOV at $t=2.2$ seconds and flows down the arteriole to nucleate on top of the existing neutrophil-platelet aggregate $(t=4.4$ seconds), resulting in the formation of a large aggregate composed of 5 neutrophils and a few platelets (dotted white circle) that completely occludes the arteriolar bottleneck. The times displayed are relative to the selected frames. Neutrophils are shown in red, platelets in green, pulmonary microcirculation in purple. Asterisks denote alveoli. Thin white arrows mark the direction of blood flow within the feeding arterioles. The diameters of the arterioles shown in A and B are $26 \mu \mathrm{m}$ and $30 \mu \mathrm{m}$, respectively. Scale bars: $20 \mu \mathrm{m}$. See Supplemental Videos 11 and 14 for the complete qFILM time series corresponding to $\mathbf{A}$ and $\mathbf{B}$.

1A, Supplemental Figure 3, and Supplemental Videos 3 and 4). Unlike SCD mice, control mice administered $0.1 \mu \mathrm{g} / \mathrm{kg}$ IV LPS had a majority of FOVs free of pulmonary vaso-occlusions, as shown in Figure 1B and Supplemental Figure 3A. We also assessed whether these vaso-occlusions blocked blood flow in arterioles. Supplemental Figure 4 shows 3 separate representative examples of pulmonary vaso-occlusions in SCD mice, which demonstrate that the occlusion of arteriolar bottlenecks by neutrophil-platelet aggregates results in a loss of blood flow evident by the absence of the vascular dye (purple fluorescence) immediately downstream of the aggregates. Supplemental Figure 5 and Supplemental Videos 5 and 6 also show another 3 representative examples of pulmonary vaso-occlusions in SCD mice, which again demonstrates that the 


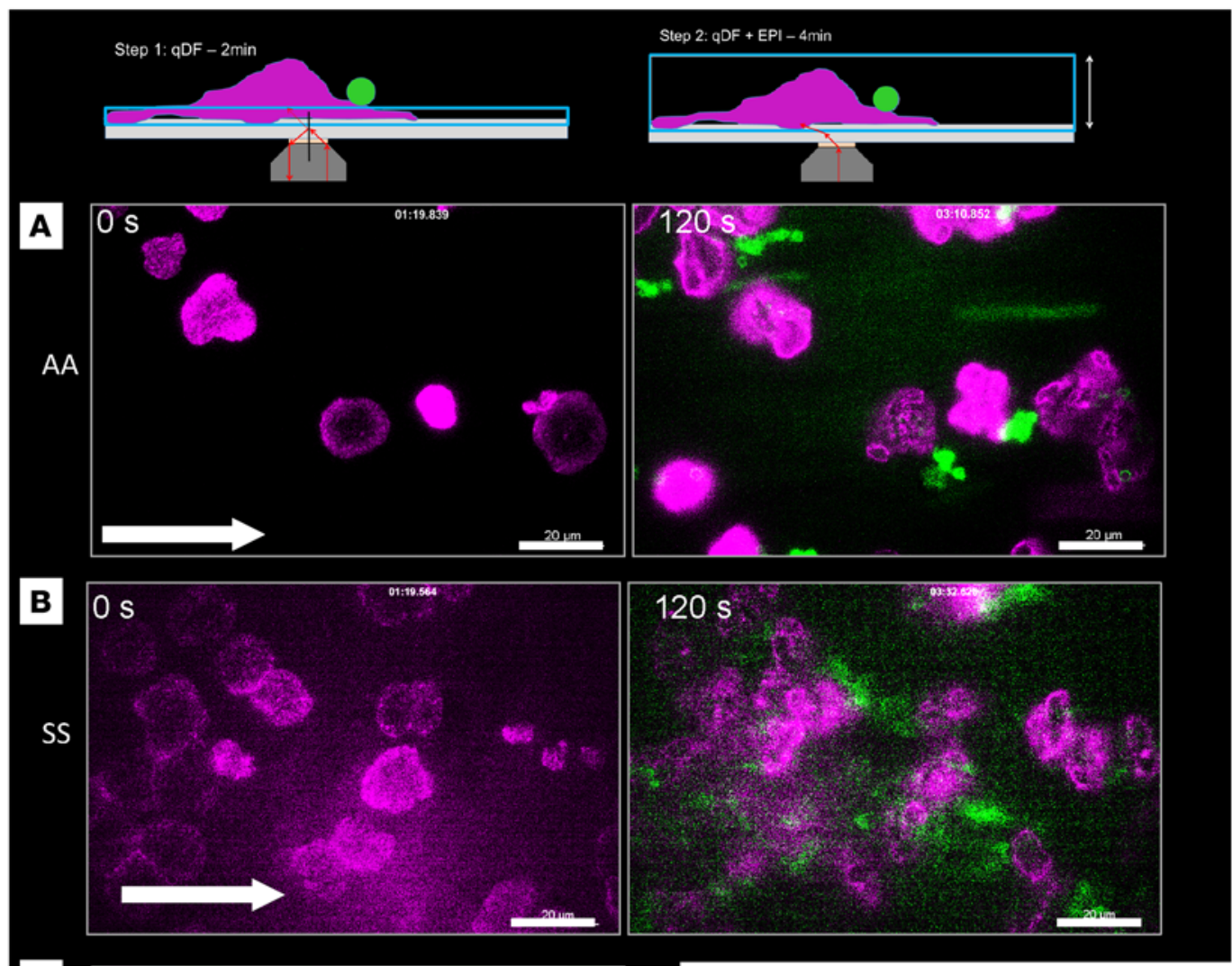

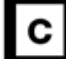
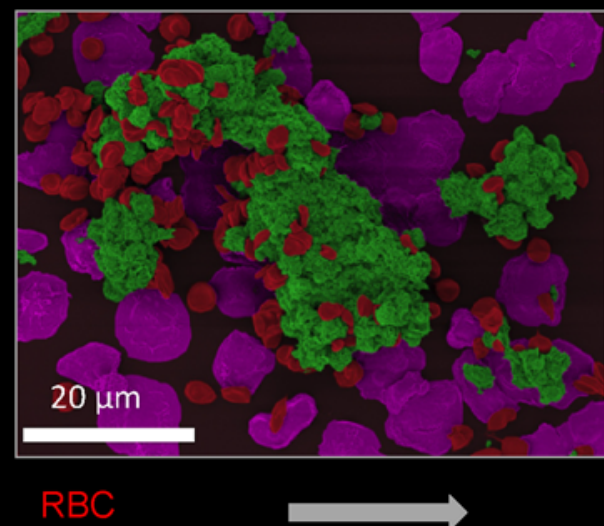

Platelet Neutrophil

\section{Direction of flow}

D

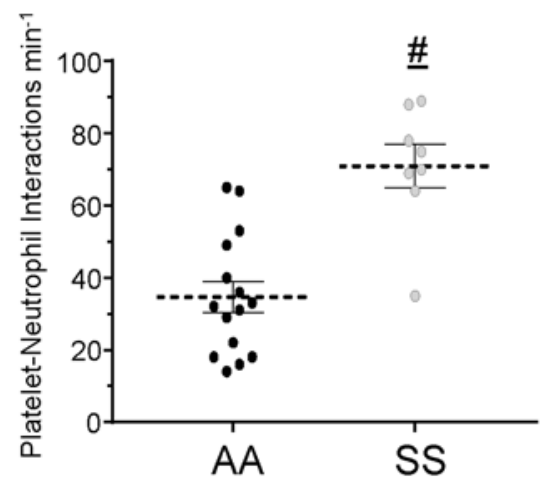

E

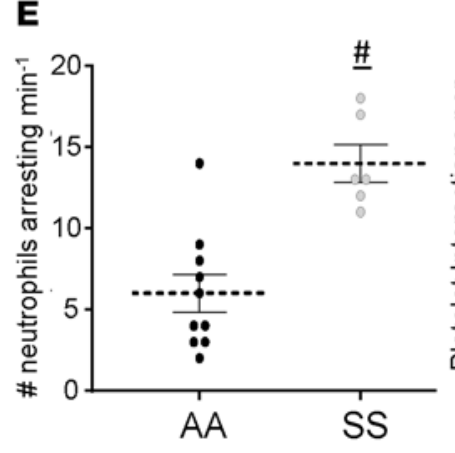

$\mathbf{F}$
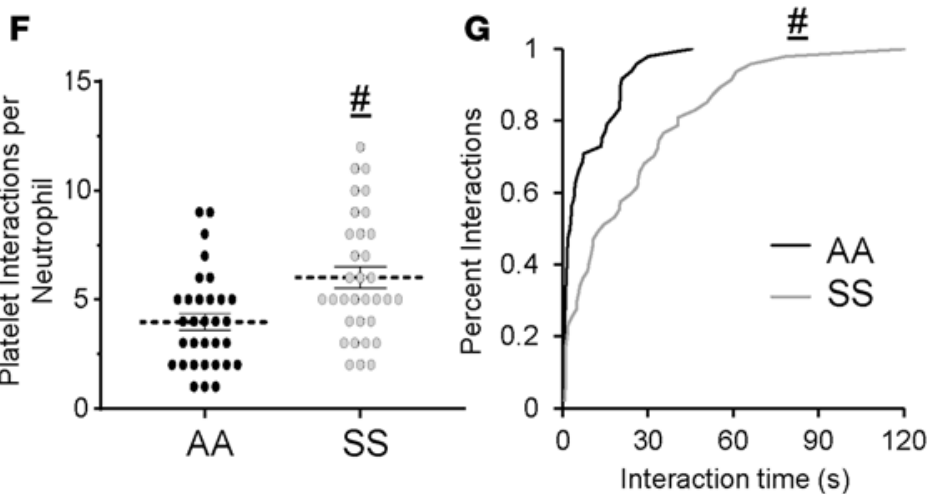
Figure 3. Neutrophil-platelet aggregation is higher in steady state sickle cell disease (SCD) human blood. African American control (AA) and steady-state SCD (SS) human whole blood was perfused through micro-channels presenting P-selectin, ICAM-1, and IL-8, and interactions of platelets with arrested neutrophils were monitored using quantitative microfluidic fluorescence microscopy (qMFM). (A and B) qMFM images of the same field of view (FOV) at 2 different time points ( 0 and 120 seconds) showing freely flowing platelets interacting with arrested neutrophils in control (A) and SCD (B) blood. Schematic above each column shows the qMFM step ( 1 or 2 ) used to acquire the images in that column. Step 1 of qMFM was used to visualize the footprints of arresting neutrophils (purple) at $t=0$ seconds, and step 2 was used to visualize nucleation of freely flowing platelets (green) on top of arrested neutrophils (purple) at $t=120$ seconds. The complete time series is shown in Supplemental Videos 17 and 18, respectively. White arrows denote the direction of blood flow. (C) Pseudocolored scanning electron micrograph showing platelets (green) nucleated on top of arrested neutrophils (purple) in SCD blood. Erythrocytes (red) are sequestered within the neutrophil-platelet aggregates. Arrow denotes the direction of flow. Scale bars: $20 \mu \mathrm{m}$. (D) Platelet interactions with arrested neutrophils shown as total interactions per minute in a FOV. (E) Total number of neutrophils arresting per minute in a FOV. (F) Platelet interactions with arrested neutrophils shown as the number of platelet interaction events per arrested neutrophil over a 2-minute observation period in a FOV. (C) Lifetime of platelet-neutrophil interactions shown as a cumulative probability distribution. The median lifetime was 2 seconds (control) and 14 seconds (SCD). A-E are representative of 6 experiments with 3 control and 3 SCD patients. $\mathbf{F}$ and $\mathbf{G}$ are representative of 8 experiments with 4 control and 4 SCD patients. Data in $\mathbf{D}-\mathbf{F}$ represent mean $\pm \mathrm{SEM}$; means were compared using Student's $t$ test. Distributions in $\mathbf{G}$ were compared using the nonparametric Kruskal-Wallis $H$ test. Each data point in $\mathbf{D}$ and $\mathbf{E}$ represents a single FOV. Observations were made using multiple FOVs in individual experiments. Each data point in $\mathbf{F}$ represents a single neutrophil. ${ }^{P} P<0.05$ when comparing control with SCD. Wall shear stress 6 dyn $/ \mathrm{cm}^{2}$.FOV: $\sim 14,520 \mu \mathrm{m}^{2}$. See Supplemental Methods for details.

occlusion of arteriolar bottlenecks by neutrophil-platelet aggregates results in the blockage of blood flow, evident by the back-and-forth pulsation of erythrocytes (green) immediately upstream of the aggregates or stationary erythrocytes immediately downstream of the aggregates.

Vaso-occlusions were assessed in randomly selected multiple (5 to 10) FOVs for every mouse and quantified using 4 parameters: average number of pulmonary vaso-occlusions per FOV, percent of FOVs with pulmonary vaso-occlusions, cellular composition of pulmonary vaso-occlusions, and area of pulmonary vaso-occlusions (see Supplemental Methods). SCD mice administered $0.1 \mu \mathrm{g} / \mathrm{kg}$ IV LPS had a significantly increased average number of pulmonary vaso-occlusions per FOV compared with SCD mice administered IV saline and control mice administered $0.1 \mu \mathrm{g} / \mathrm{kg}$ IV LPS (Figure 1C). The average number of pulmonary vaso-occlusions was not significantly different between control mice administered $0.1 \mu \mathrm{g} / \mathrm{kg}$ IV LPS or saline. Interestingly, the average number of pulmonary vaso-occlusions per FOV in control mice administered $0.1 \mu \mathrm{g} / \mathrm{kg}$ IV LPS appeared similar to that in SCD mice administered IV saline. SCD mice administered $0.1 \mu \mathrm{g} / \mathrm{kg}$ IV LPS had a significantly higher percentage of FOVs with pulmonary vaso-occlusions compared with SCD mice given IV saline (Figure 1D). The percentage of FOVs with pulmonary vaso-occlusions after administration of $0.1 \mu \mathrm{g} / \mathrm{kg}$ IV LPS also trended higher in SCD compared with control mice, but the difference was not statistically significant. Previously, higher doses of IV LPS have been shown to induce vascular congestion in the lungs of even WT mice (24). Therefore, we tested whether a stronger inflammatory challenge would also induce pulmonary vaso-occlusion in control mice. As expected and unlike $0.1 \mu \mathrm{g} /$ $\mathrm{kg}$ IV LPS, the frequency and size of neutrophil-platelet aggregates blocking the arteriolar bottlenecks at a 10 -fold-higher dose of $1 \mu \mathrm{g} / \mathrm{kg}$ IV LPS was significantly large and not different between control and SCD mice (Supplemental Figures 6 and 7). This finding suggests that unlike $1 \mu \mathrm{g} / \mathrm{kg}$ IV LPS, which promotes pulmonary vaso-occlusion in both control and SCD mice, the 10 -fold-smaller dose of $0.1 \mu \mathrm{g} / \mathrm{kg}$ IV LPS is innocuous to control mice but potent enough to promote pulmonary vaso-occlusion in SCD mice.

Cellular composition and size of pulmonary vaso-occlusions. Pulmonary vaso-occlusions were classified into the following three categories based on their cellular composition: (a) neutrophil vaso-occlusions (red arrow in the schematic shown in Figure 1E) comprising predominantly neutrophils (red) with few adhered platelets (green); (b) platelet vaso-occlusions (green arrow in Figure 1E) comprising predominantly platelets with few adhered neutrophils; and (c) occasionally observed other vaso-occlusions (not shown in the schematic) comprising predominantly other cell types with few adhered neutrophils and/or platelets. A qFILM image from a SCD mouse administered $0.1 \mu \mathrm{g} / \mathrm{kg}$ IV LPS is shown in Figure 1F. Supplemental Video 7 (same FOV as shown in Figure 1F) highlights that all 4 arteriolar bottlenecks are occluded by either neutrophil (red arrows) or platelet (green arrows) vaso-occlusions, which restrict the blood flow. Two of these vaso-occlusions (circles with longer dashes in Figure 1F) were magnified to highlight a neutrophil vaso-occlusion (Figure 1G) with several neutrophils (red) bound to a few platelets (green) and a platelet vaso-occlusion (Figure 1H) with mainly platelets bound to a single neutrophil (white arrow). As shown in Figure 1I, SCD mice administered $0.1 \mu \mathrm{g} / \mathrm{kg}$ IV LPS had significantly greater neutrophil and platelet vaso-occlusions compared with control mice administered $0.1 \mu \mathrm{g} / \mathrm{kg}$ IV LPS. Neutrophil vaso-occlusions were also significantly increased in $0.1 \mu \mathrm{g} /$ $\mathrm{kg}$ IV LPS SCD mice compared with IV saline SCD mice. The neutrophil vaso-occlusions appeared to be the most widely occurring vaso-occlusions in the pulmonary microcirculation. 

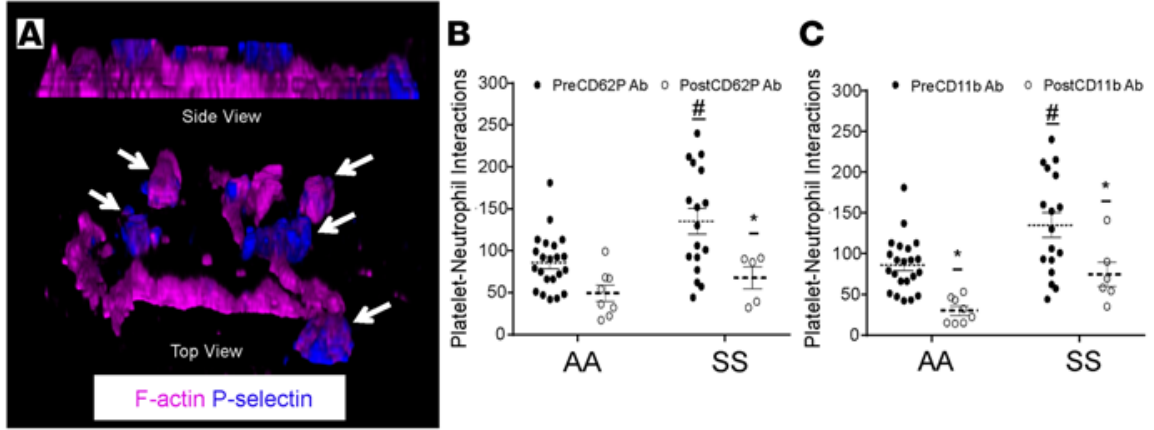

D

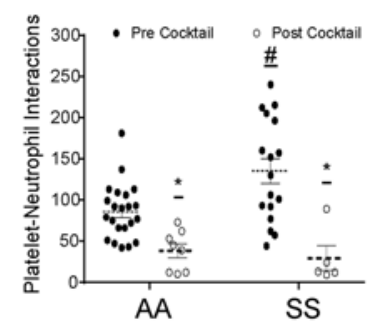

E

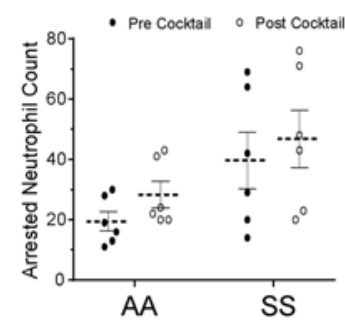

F

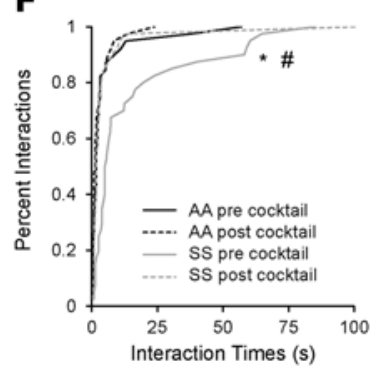

Figure 4. Neutrophil-platelet aggregation in sickle cell disease (SCD) human blood is platelet P-selectin dependent. African American control (AA) and steady-state SCD (SS) human whole blood with or without addition of function blocking mAbs against P-selectin and/or Mac-1 was perfused through micro-channels presenting P-selectin, ICAM-1, and IL-8, and interactions of platelets with arrested neutrophils were monitored using quantitative microfluidic fluorescence microscopy (qMFM). (A) Structured illumination micrograph of platelets nucleated on an arrested neutrophil in SCD blood. F-actin (purple) can be seen throughout the outer ring (lamellipodium) of the arrested neutrophil and within the nucleating platelets. CD62P (blue) is expressed primarily on the platelets. Platelets are marked with white arrows. See also Supplemental Video 19. (B) Effect of platelet P-selectin inhibition on total platelet interactions with arrested neutrophils. (C) Effect of Mac-1 inhibition on total platelet interactions with arrested neutrophils. ( $\mathbf{D}$ and $\mathbf{E}$ ) Effect of simultaneous inhibition of platelet P-selectin and neutrophil Mac-1 on (D) total platelet interactions with arrested neutrophils and (E) total number of arrested neutrophils. (F) Effect of simultaneous inhibition of P-selectin and Mac-1 on the lifetime of platelet-neutrophil interactions. The median lifetime was $\sim 1.8$ seconds (control before Ab cocktail treatment [AA pre cocktail], control after cocktail [AA post cocktail], and SCD after cocktail [SS post cocktail]) vs. 5 seconds (SCD before cocktail [SS pre cocktail]). The data for AA pre and SS pre shown in $\mathbf{F}$ are not the same as the data in Figure 3G; the 2 sets of data were generated using a separate set of experiments. Wall shear stress: $6 \mathrm{dyn} / \mathrm{cm}^{2}$. FOV: $\sim 14,520 \mu \mathrm{m}^{2}$. B-D representative of 10 experiments with 4 control and 5 SCD patients; $\mathbf{E}$ and $\mathbf{F}$ representative of 6 experiments with 3 control and 3 SCD patients. Data represent mean \pm SEM. Means in B-E were compared using Student's $t$ test with Bonferroni correction. Interaction times in $\mathbf{F}$ were compared using the nonparametric Kruskal-Wallis $H$ test. Each data point in B-E (black circles, pre-Ab treatment; white circles, post-Ab treatment) represents a single field of view (FOV), and observations were made using multiple FOVs in individual experiments. ${ }^{\#} P<0.05$ when comparing control with $\mathrm{SCD}$; ${ }^{*} P<0.05$ when comparing pre- and post-Ab treatment. Wall shear stress: $6 \mathrm{dyn} / \mathrm{cm}^{2}$. FOV: $\sim 14,520 \mu \mathrm{m}^{2}$.

To validate this observation, we also conducted flow cytometry analysis of digested whole lung cell suspensions to estimate the magnitude of neutrophil-platelet aggregation in control and SCD mice following administration of IV saline or 0.1 $\mu \mathrm{g} / \mathrm{kg}$ IV LPS (Supplemental Figure 8). As shown in Supplemental Figure 8, SCD mice had a higher number of neutrophil-platelet aggregates sequestered in the lung compared with control mice following administration of $0.1 \mu \mathrm{g} / \mathrm{kg}$ IV LPS. We next hypothesized that the low dose of $0.1 \mu \mathrm{g} /$ $\mathrm{kg}$ IV LPS mediates pulmonary vaso-occlusion by selectively triggering the formation of large neutrophil-platelet aggregates in SCD mice. To test this, we estimated the area of the pulmonary vaso-occlusions in all qFILM images using dashed ellipses, similar to those shown in Figure 1E (see Supplemental Methods for details). The average number of large pulmonary vaso-occlusions (area $>1,000 \mu \mathrm{m}^{2}$ ) following $0.1 \mu \mathrm{g}$ / $\mathrm{kg}$ IV LPS was significantly increased in SCD compared with control mice (Figure $1 \mathrm{~J})$, suggesting that the formation of large neutrophil-platelet aggregates contributes to the blockage of arteriolar bottlenecks. We found that erythrocytes were also present in a small number of neutrophil-platelet aggregates. Supplemental Figure 9 and Supplemental Videos 8-10 show 6 separate representative examples of pulmonary vaso-occlusions in SCD mice, which reveal the presence of erythrocytes (green) inside and around the neutrophil (red)-platelet (blue) aggregates. Erythrocytes have been shown to promote vaso-occlusion in the cremaster venules of SCD mice (8), and their role in lung vaso-occlusion will be further elucidated in future studies.

Microembolic or in situ nucleated large aggregates occlude arteriolar bottlenecks. Next,
nucleated in situ or arrive as a microembolic we asked whether the large neutrophil-platelet aggregates are nucleated in situ or arrive as a microembolic
aggregate within the pulmonary arteriole to mediate pulmonary vaso-occlusion. As shown in Figure 2 , qFILM revealed that either process can contribute to the initiation of pulmonary vaso-occlusion in SCD mice administered $0.1 \mu \mathrm{g} / \mathrm{kg}$ IV LPS. As shown in Figure $2 \mathrm{~A}$ and Supplemental Video 11, a microembolic aggregate composed of several neutrophils and a few platelets enters the FOV at $t=1.5$ seconds and travels down the arteriole to block the bottleneck at the junction of arterioles and capillaries by $t=16 \mathrm{sec}$ onds. This observation is further supported by Supplemental Figure 10, which shows additional examples of neutrophil-platelet microemboli in the pulmonary arterioles. Circulating neutrophil-platelet embolic aggregates are shown transiting through the pulmonary arterioles (Supplemental Video 12) and stopping within the arteriolar bottleneck to contribute to pulmonary vaso-occlusions (Supplemental Video 13). Figure 2B and Supplemental Video 14 demonstrate a neutrophil-platelet aggregate of 3 neutrophils and a 
A

SS

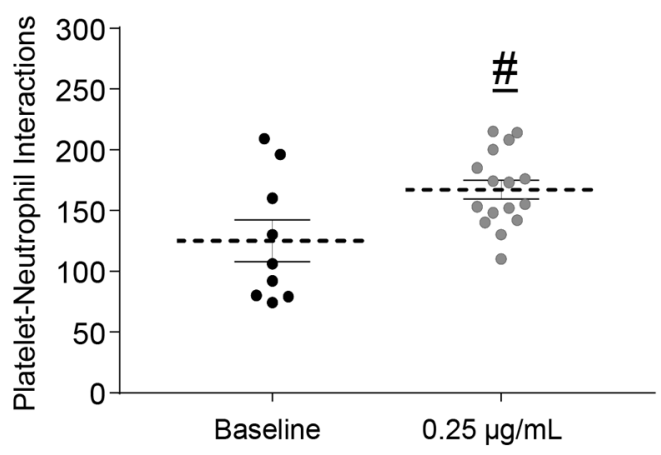

LPS Concentration

C

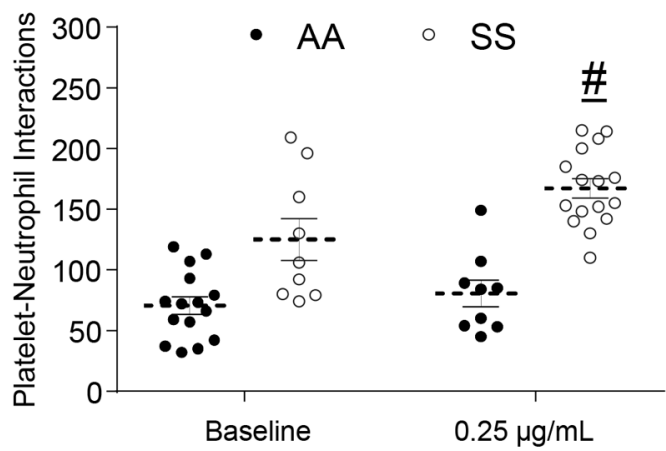

LPS Concentration

E

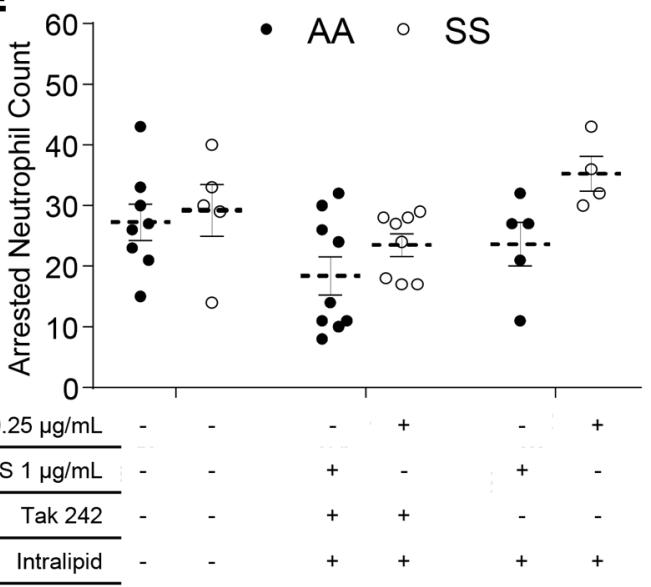

B

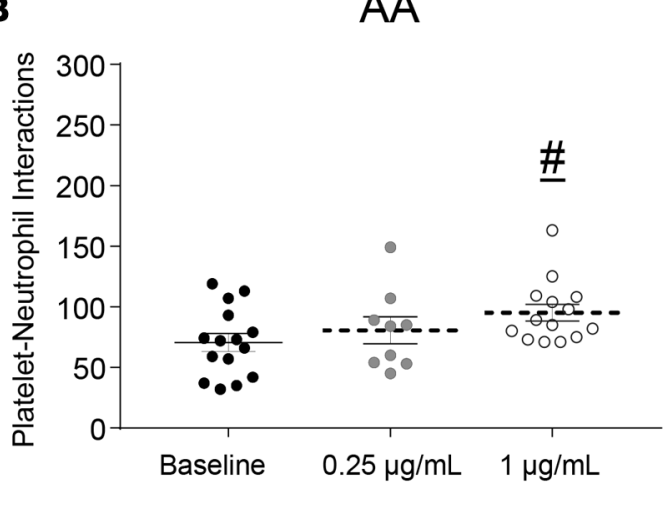

LPS Concentration

D

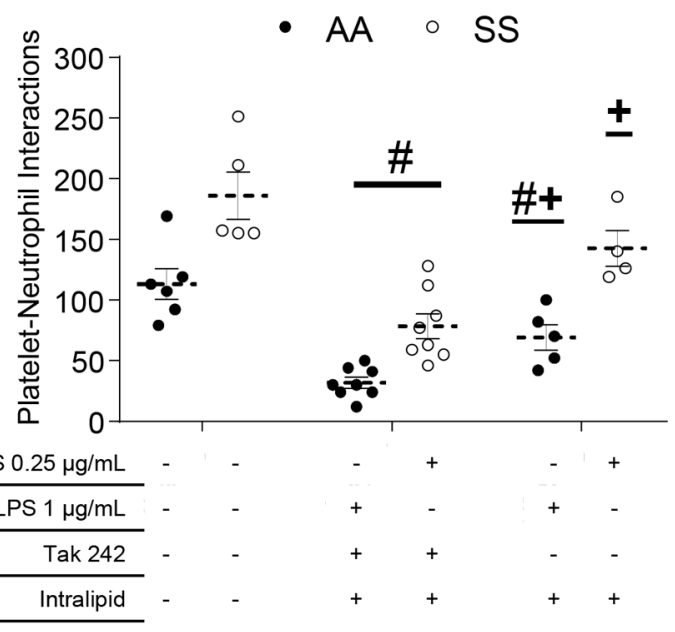

Figure 5. LPS promotes neutrophil-platelet aggregation in sickle cell disease (SCD) human blood. African American control (AA) or steady-state SCD (SS) human whole blood with or without LPS pretreatment was perfused through micro-channels presenting P-selectin, ICAM-1, and IL-8; and neutrophil-platelet interactions were monitored using quantitative microfluidic fluorescence microscopy (qMFM) over a 2-minute period. (A) Total platelet interactions with arrested neutrophils in SCD (steady state) whole blood with or without pretreatment with $0.25 \mu \mathrm{g} / \mathrm{ml}$ of LPS. Representative of 6 experiments with 6 SCD subjects. (B) Total platelet interactions with arrested neutrophils in control human blood with or without pretreatment with 0.25 or $1 \mu \mathrm{g} / \mathrm{ml}$ LPS. Representative of 6 experiments with 5 control subjects. (C) Comparison of total platelet-neutrophil interactions following pretreatment of control and SCD blood with $0.25 \mu \mathrm{g} / \mathrm{ml}$ LPS. Representative of 8 experiments with 3 control and 5 SCD subjects. (D and E) Effect of TAK-242 and/or intralipid (vehicle) pretreatment on (D) the total number of platelet interactions with arrested neutrophils and (E) total number of arrested neutrophils over a 2-minute observation period in 0.25 or $1 \mu \mathrm{g} / \mathrm{ml}$ LPS-treated SCD or control human blood, respectively. TAK- 242 was added to the blood ( $50 \mu \mathrm{g} / \mathrm{ml}$ ) and incubated for 5 minutes. After 5 minutes, LPS was added to the blood and incubated for 10 minutes before perfusion through the micro-channels. Representative of 6 experiments with 3 control and 3 SCD subjects. Data represent mean \pm SEM. ${ }^{*} P<0.05$ when comparing with baseline; ${ }^{+} P<0.05$ when comparing with TAK-242. Means were compared using Student's $t$ test with Bonferroni correction. Each data point represents a single field of view (FOV), and observations were made over multiple FOVs in some experiments. See Supplemental Methods for details. Wall shear stress: $6 \mathrm{dyn} / \mathrm{cm}^{2}$. FOV: $\sim 4,520 \mu \mathrm{m}^{2}$. 
A

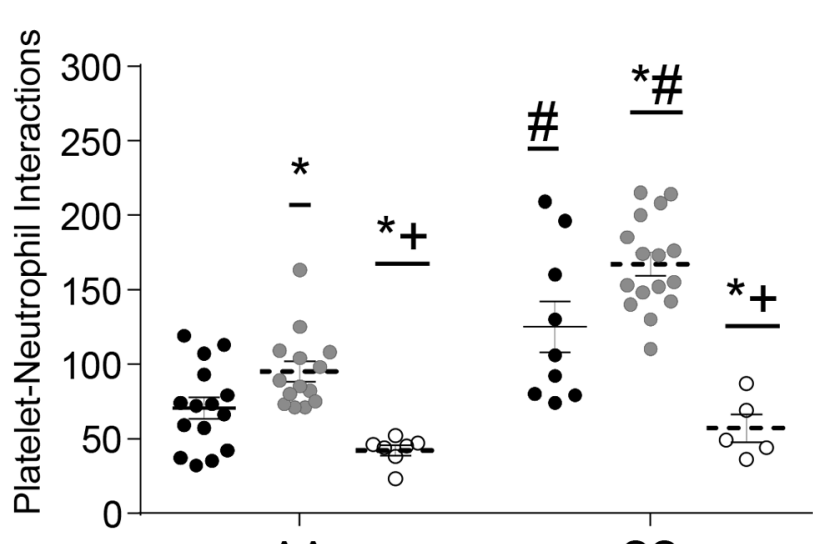

C

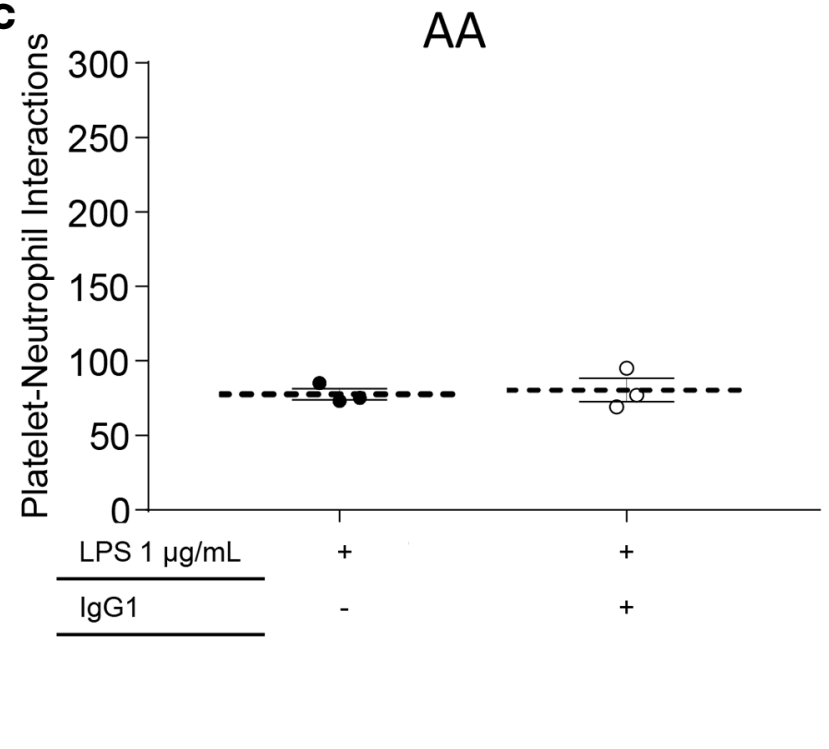

B

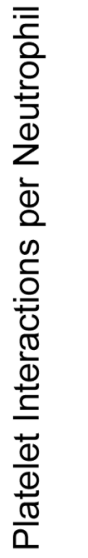
AA \begin{tabular}{lllllll}
$\frac{\text { LPS } 0.25 \mu \mathrm{g} / \mathrm{mL}}{\mathrm{LPS} 1 \mu \mathrm{g} / \mathrm{mL}}$ & - & - & - & - & + & + \\
\hline Ab Cocktail & + & + & - & - & - \\
\hline & - & + & - & - & +
\end{tabular}

D

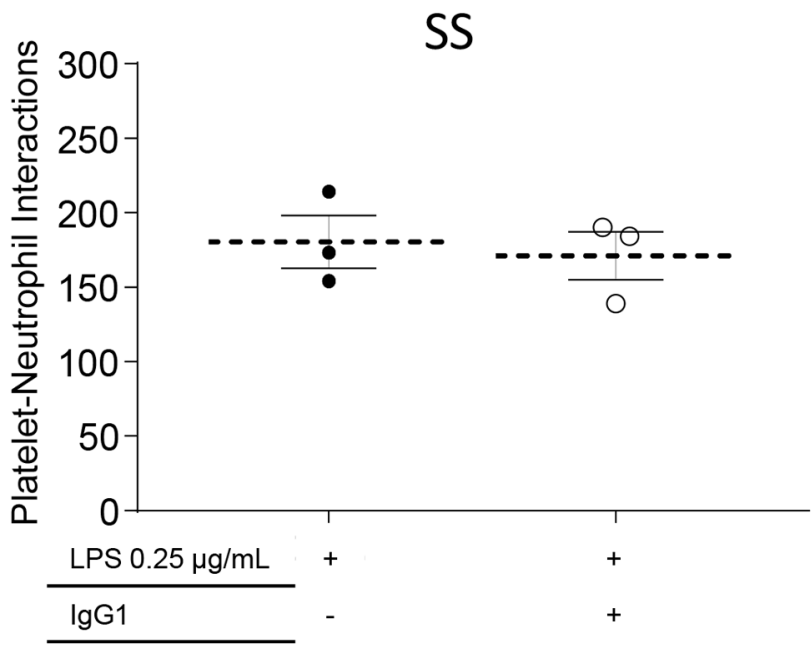

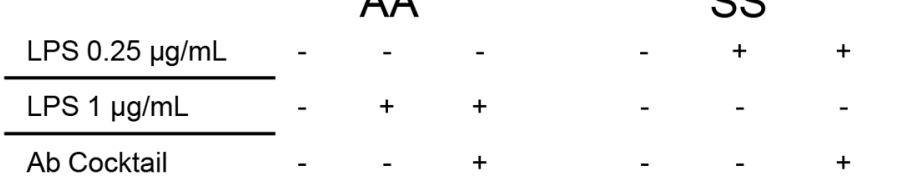

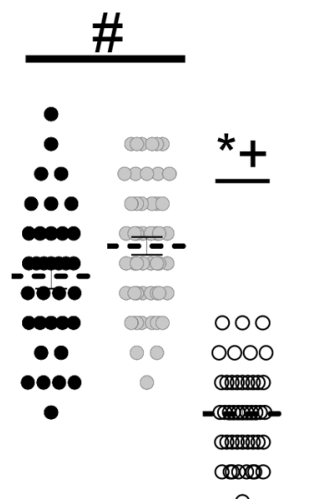

SS

Figure 6. LPS-induced neutrophil-platelet aggregation is P-selectin and Mac-1 dependent. African American control (AA) or steady -state sickle cell disease (SS) human whole blood with or without LPS pretreatment and with or without addition of a cocktail of P-selectin and Mac- 1 function-blocking mAbs was perfused through micro-channels presenting P-selectin, ICAM-1, and IL-8; and neutrophil-platelet interactions were monitored in a field of view (FOV) using quantitative microfluidic fluorescence microscopy (qMFM) over a 2-minute period. LPS was added to the blood and incubated at room temperature for 10 minutes before perfusion through the micro-channels. Flow was stopped after 2 minutes of perfusion, and function-blocking antibodies were added to the blood. Flow was resumed, and observations were made for another 2 minutes. (A and B) Effect of simultaneous inhibition of platelet P-selectin and neutrophil Mac-1 on (A) total platelet interactions with arrested neutrophils and (B) number of platelet interaction events per arrested neutrophil in control or SCD human blood with or without pretreatment with LPS $(0.25$ or $1 \mu \mathrm{g} / \mathrm{ml})$. ${ }^{*} P<0.05$ when compared with baseline; ${ }^{\#} P<0.05$ when comparing control with SCD; ${ }^{+} P<0.05$ when comparing LPS with Ab treatment. Representative of 8 experiments with 4 control and 4 SCD subjects. (C and $\left.\mathbf{D}\right)$ No effect of isotype IgG control Ab treatment on the total number of platelet interactions with arrested neutrophils in (C) $1 \mu \mathrm{g} / \mathrm{ml}$ LPS-treated control and (D) 0.25 $\mu \mathrm{g} / \mathrm{ml}$ LPS-treated SCD human blood. Representative of 5 experiments done with 2 control and 3 SCD subjects. Data represent mean \pm SEM. Means were compared using Student's $t$ test with Bonferroni correction for multiple comparisons. Each data point represents a single field of view (FOV), and observations were made over multiple FOVs in individual experiments. See Supplemental Methods for details. Wall shear stress: $6 \mathrm{dyn} / \mathrm{cm}^{2}$. FOV: $\sim 14,520 \mu \mathrm{m}^{2}$.

few platelets partially occluding the arteriolar bottleneck at $t=0$ seconds. The fourth and fifth neutrophil enter the FOV in tandem and nucleate on top of the existing aggregate at $t=2$ and 4 seconds, respectively, to form an aggregate large enough to completely occlude the arteriolar bottleneck. Supplemental Figure 11 shows additional examples demonstrating in situ nucleation of neutrophil-platelet aggregates 
Table 1. Clinical characterization of human subjects

\begin{tabular}{|c|c|c|}
\hline & Control & SCD \\
\hline Male/Female & $4 / 2$ & $5 / 19$ \\
\hline Hemoglobin (g/dl) & $12.46(11.2 ; 14.4 ; 12.1)$ & 9.06 (6.6; 11.2; 9.3) \\
\hline White blood cells (k/dl) & $4.62(2.5 ; 8.68 ; 4.43)$ & 9.88 (3; 15.72; 11.05) \\
\hline$\%$ Neutrophils & NM & $55.2(1.7 ; 77.2 ; 57.6)$ \\
\hline Neutrophil count $(\mathrm{k} / \mu \mathrm{l})$ & NM & $6.89(1.7 ; 28 ; 6.3)$ \\
\hline$\% \mathrm{HbS}$ & NM & $58.32(5.9 ; 80.9 ; 64.6)$ \\
\hline \multicolumn{3}{|l|}{ Genotypes } \\
\hline SS & $0 / 6$ & $23 / 24$ \\
\hline $\mathrm{S} / \beta^{0}$ & $0 / 6$ & $1 / 24$ \\
\hline AS & $1 / 6$ & $0 / 24$ \\
\hline
\end{tabular}

Data show mean (minimum; maximum; median) except for sex and genotypes. SCD, sickle cell disease; SS, sickle cell anemia; S/ $\beta^{0}$, sickle $/ \beta^{0}$ thalassemia; AS, sickle cell trait; AA, healthy control; \%HbF, \% fetal hemoglobin; \%HbS, \% sickle hemoglobin; NM, not measured.

in pulmonary arterioles. In Supplemental Videos 15 and 16, single circulating neutrophils adhere to preexisting aggregates of 3 neutrophils and a few platelets in the arteriolar bottleneck to promote pulmonary vaso-occlusion.

Neutrophil-platelet aggregation is higher in steady-state human SCD blood under flow. In order to explore the relevance of observations in the transgenic SCD mouse, normal control and SCD human blood was collected in heparin and perfused at a physiological shear stress of $6 \mathrm{dyn} / \mathrm{cm}^{2}(25)$ through microfluidic micro-channels presenting a combination of P-selectin, ICAM-1, and IL-8. See Table 1 for the clinical characterization of human subjects. qMFM was used to visualize neutrophil-platelet aggregation at the level of single cell-cell interactions (19) (see Methods for details). The magnitude of neutrophil-platelet aggregate formation was assessed based on the ability of free-flowing platelets to interact with arrested neutrophils, which was quantified using 3 parameters: total number of platelet-neutrophil interactions; number of platelets that interact per arrested neutrophil; and the lifetimes of individual interactions of platelets with arrested neutrophils over a 2-minute observation in a FOV of size of $\sim 14,520 \mu \mathrm{m}^{2}$. When control or steady-state SCD human blood was perfused through micro-channels presenting P-selectin, ICAM-1, and IL-8, neutrophils were observed to roll, arrest, crawl, and interact with freely flowing platelets (Figure 3, A and B, and Supplemental Videos 17 and 18). A snapshot of the same FOV at two different time points 2 minutes apart is shown for control and SCD human blood in Figure 3, A and B, respectively. Comparison of Figure 3A with Figure $3 \mathrm{~B}$ at the time point of 2 minutes reveals that platelet interactions with arrested neutrophils led to the formation of large neutrophil-platelet aggregates, which appeared to be more pronounced in SCD than control human blood. Neutrophil-platelet aggregates in SCD blood were fixed under flow and visualized using scanning electron microscopy (SEM). A pseudo-colored scanning electron micrograph of large neutrophil-platelet aggregates comprising platelets (green) nucleated on arrested neutrophils (purple) is shown in Figure 3C. Erythrocytes (pseudo-colored red) can be seen sequestered within these large neutrophil-platelet aggregates (Figure 3C). Quantitative analysis of several time-series of qMFM images revealed that the total number of platelet-neutrophil interactions occurring per minute in a FOV were 2-fold higher in steady-state SCD than control human blood (Figure 3D). Similar to what we have reported previously (19), we found that the number of neutrophils arresting per minute in the same FOV was also 2-fold higher in steady-state SCD than control human blood (Figure 3E). To test whether the significantly higher number of platelet-neutrophil interactions was a direct consequence of a higher number of arresting neutrophils, we estimated the number of platelet-neutrophil interactions per arrested neutrophil. As shown in Figure 3F, 1.5-fold more platelets were observed to interact per neutrophil over a 2-minute observation period in steady-state SCD compared with control human blood, thus excluding a major role of higher neutrophil numbers in driving increased platelet-neutrophil interactions. Interestingly, when we measured the lifetime of individual platelet-neutrophil interactions and plotted it as a cumulative probability distribution (Figure 3G), the lifetimes were significantly longer in SCD (median lifetime $\sim 14$ seconds) than control (median lifetime $\sim 2$ 

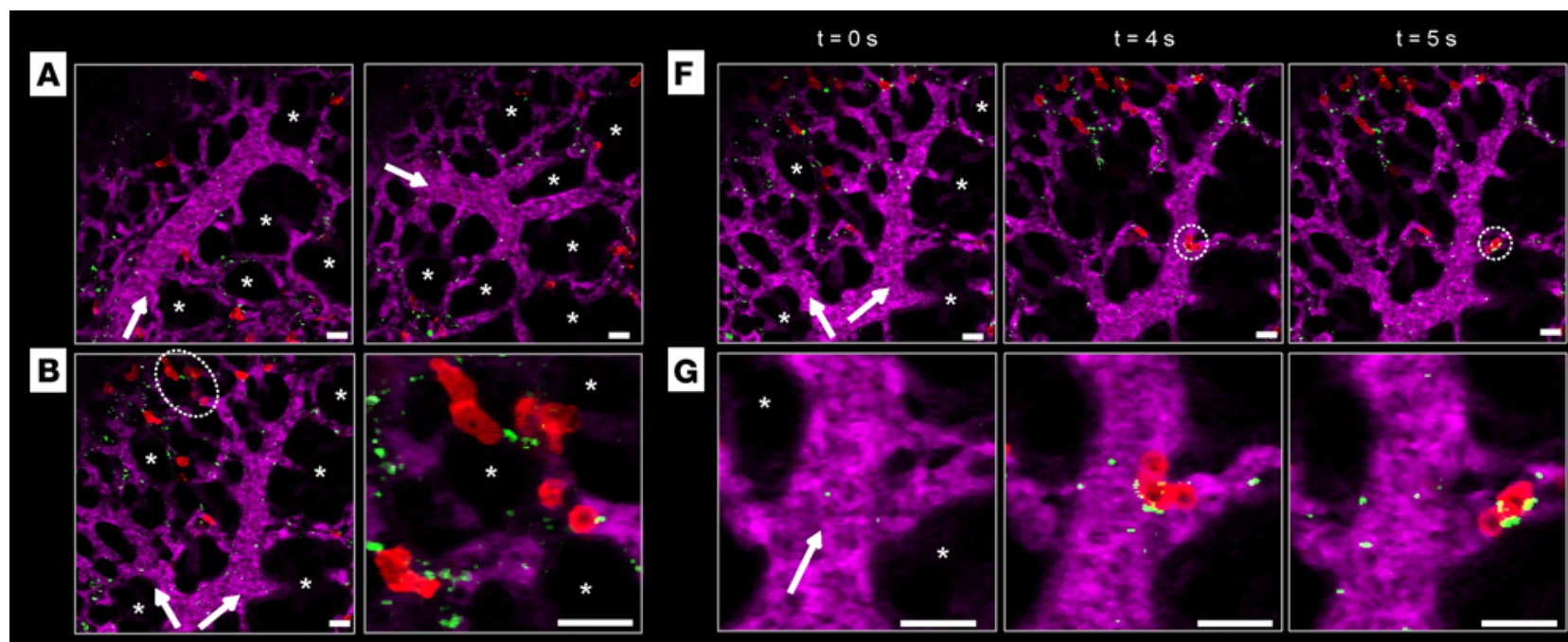

C

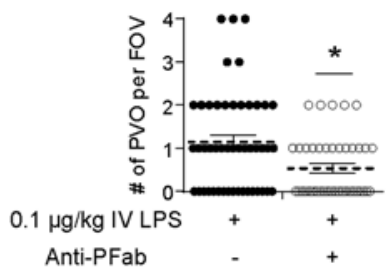

D

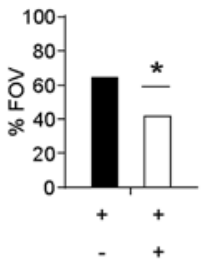

E

$0.1 \mu \mathrm{g} / \mathrm{kg}$ IV LPS SCD $0.1 \mu \mathrm{g} / \mathrm{kg}$ IV LPS + anti-PFab SCD

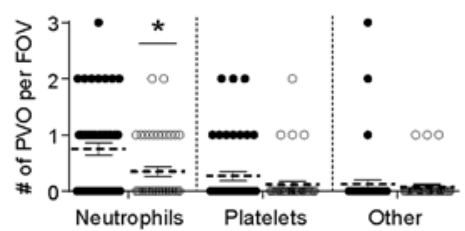

H

- $0.1 \mu \mathrm{g} / \mathrm{kg}$ IV LPS SCD

$0.1 \mu \mathrm{g} / \mathrm{kg}$ IV LPS + anti-PFab SCD

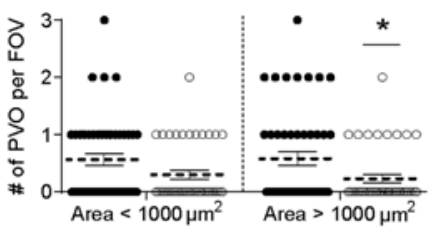

Figure 7. P-selectin blockade ameliorates LPS-induced pulmonary arteriole microembolism in SCD mice. Sickle cell disease (SCD) mice were injected intravenously (IV) with $0.1 \mu \mathrm{g} / \mathrm{kg}$ LPS ( $n=5$ mice; 48 fields of view [FOVs]) or $0.1 \mu \mathrm{g} / \mathrm{kg}$ IV LPS + anti-P-selectin mAb Fab fragments ( $n=3$ mice; 43 FOVs). Arterioles were imaged 2-2.5 hours after IV LPS or IV LPS + anti-P-selectin Fab fragments using quantitative fluorescence intravital lung microscopy (qFILM). (A and B) qFILM images of 3 FOVs from a SCD mouse administered IV LPS + anti-P-selectin Fab fragments. (A) A majority (58\%) of FOVs show no pulmonary vaso-occlusions. Supplemental Video 20 shows the FOV in A (right panel). (B) Anti-P-selectin Fab fragments attenuated LPS-induced arteriolar pulmonary vaso-occlusions. A small aggregate of neutrophils (red) and platelets (green) blocks the arteriolar bottleneck (white dotted circle). Magnified view of vaso-occlusion is shown to the right. Pulmonary microcirculation is shown in purple. Asterisks denote alveoli. White arrows mark the direction of blood flow. Average diameter of arterioles in $\mathbf{A}$ and $\mathbf{B}: 30 \pm 2 \mu \mathrm{m}$. Scale bars: $20 \mu \mathrm{m}$. (C-H) Data from SCD mice administered $0.1 \mu \mathrm{g} /$ kg IV LPS are repeated from Figure 1. (C) Number of pulmonary vaso-occlusions (PVO) per FOV for SCD mice administered IV LPS (black circles) or IV LPS + anti-P-selectin Fab fragments (white circles). Size of FOV: $\sim 67,600 \mu \mathrm{m}^{2}$. (D) Percent FOVs with pulmonary vaso-occlusions. (E) Number of pulmonary vaso-occlusions per FOV classified by cellular composition. (F) qFILM images of one FOV at 3 different time points in a SCD mouse administered IV LPS + anti-P-selectin Fab fragments. A neutrophil-platelet aggregate (white dotted circles) arrives and disintegrates within a pulmonary arteriole. (G) Magnified view of neutrophil-platelet aggregate in $\mathbf{F} . t=0 \mathrm{~s}$ shows the arteriole before the aggregate arrives. At $t=4$ seconds, the neutrophil-platelet aggregate appears and quickly enters the arteriolar bottleneck. By $t=5$ seconds, one neutrophil has detached and traversed farther into the capillary. Diameter of arteriole in $\mathbf{F}$ and $\mathbf{G}: 28 \mu \mathrm{m}$. Scale bars: $20 \mu \mathrm{m}$. Supplemental Video 21 shows the FOV in $\mathbf{G}$. (H) Number of pulmonary vaso-occlusions per FOV with area $<1,000 \mu \mathrm{m}^{2}$ or $>1,000 \mu \mathrm{m}^{2}$. Average number of pulmonary vaso-occlusions per FOV, area of pulmonary vaso-occlusions, and cellular composition of pulmonary vaso-occlusions were compared using unpaired $t$ tests. Percent FOVs with pulmonary vaso-occlusions were compared using 4-fold table analyses with Bonferroni $\chi^{2}$ statistics. Data represent mean \pm SEM. ${ }^{*} P<0.05$ for $0.1 \mu \mathrm{g} / \mathrm{kg}$ IV LPS SCD vs. $0.1 \mu \mathrm{g} / \mathrm{kg}$ IV LPS + anti-PFab SCD.

seconds), suggesting that neutrophil-platelet aggregate formation under flow is more efficient in steady-state SCD than control human blood. These in vitro qMFM studies suggest that neutrophil-platelet interactions were significantly more frequent and of longer duration in steady-state SCD than control human blood flowing through micro-channels under vascular mimetic shear flow conditions.

Neutrophil-platelet aggregation in SCD human blood is platelet $P$-selectin dependent. Platelet-neutrophil interactions are known to be primarily mediated by PSGL-1 and CD11b-CD18 (Mac-1) on neutrophils binding to P-selectin and GPIb $\alpha$, respectively, on platelets $(10,26)$. PSGL-1 is constitutively expressed on neutrophils, and Mac-1 has been shown to be activated in arrested neutrophils (8). Although, GPIb $\alpha$ is constitutively expressed on platelets (26), P-selectin is stored preformed in $\alpha$-granules and translocated to the membrane for presentation only following activation by platelet agonists both in vivo and in vitro (10). To test whether 
P-selectin is present on steady-state human SCD platelets, neutrophil-platelet aggregates were fixed under flow, stained for P-selectin and F-actin, and subjected to super-resolution fluorescence 3D structured illumination microscopy (SIM). The SIM micrograph shown in Figure 4A and Supplemental Video 19 reveals that platelets nucleated on top of arrested neutrophils in steady-state SCD human blood are positive for P-selectin (blue staining). To determine whether P-selectin expression on the surface of platelets in SCD blood facilitates their interaction with arrested neutrophils, qMFM studies were conducted in the presence of a function blocking anti-P-selectin Ab using an experimental approach (described in Supplemental Methods) that selectively blocks platelet P-selectin without compromising P-selectin-dependent neutrophil rolling. Platelet P-selectin inhibition led to a significant drop in the ability of freely flowing platelets to nucleate on arrested neutrophils in steady-state SCD human blood (Figure 4B). As a next step, we tested the role of Mac-1-GPIba interaction by conducting qMFM studies in the presence of anti-CD11b function-blocking $\mathrm{Ab}$ using an experimental approach (described in Supplemental Methods) that allowed blocking neutrophil CD11b without interfering with neutrophil arrest. As shown in Figure 4C, CD11b inhibition also led to a significant reduction in platelet-neutrophil interactions in both control and steady-state SCD blood. Remarkably, a combination of P-selectin and CD11b function blocking Abs (anti-P-selectin/CD11b $\mathrm{Abs}$ ) led to a substantial drop in platelet-neutrophil interactions in both control and SCD human blood. As shown in Figure 4D, platelet-neutrophil interactions following treatment with anti-P-selectin/CD11b Abs were identical in SCD and control human blood; this was not the result of an Ab-induced neutrophil detachment from the substrate, as there was no difference in the number of arrested neutrophils before and after anti-P-selectin/CD11b Abs in either control or SCD human blood (Figure 4E). To establish that the anti-P-selectin/CD11b Ab-induced reduction in total platelet-neutrophil interactions was a direct effect of the inability of individual platelets to bind to arrested neutrophils, we assessed the effect of anti-P-selectin/CD11b Abs on the lifetime of individual platelet-neutrophil interactions (Figure 4F). As anticipated, the anti-P-selectin/CD11b Ab treatment reduced the lifetime of platelet-neutrophil interactions in SCD blood to the level observed in control blood, evidenced by the overlap in the lifetime distribution curves of control, control post-anti-P-selectin/CD11b Abs, and SCD post-anti-P-selectin/CD11b Abs (Figure 4F).

Low dose of LPS selectively augments neutrophil-platelet aggregation in SCD human blood. Identical to the findings in SCD mice in vivo, qMFM revealed that pretreatment of steady-state SCD human blood with LPS at a concentration of $0.25 \mu \mathrm{g} / \mathrm{ml}$ selectively augmented neutrophil-platelet aggregate nucleation in SCD but not control human blood, suggesting that the SCD blood had a higher sensitivity to LPS. As shown in Figure 5A, pretreatment with an LPS concentration of $0.25 \mu \mathrm{g} / \mathrm{ml}$ was potent enough to cause a significant increase in platelet-neutrophil interactions in steady-state SCD human blood. In contrast, LPS at a final concentration of $0.25 \mu \mathrm{g} / \mathrm{ml}$ did not result in any increase in platelet-neutrophil interactions in control human blood, but rather a 4 -fold higher concentration $(1 \mu \mathrm{g} / \mathrm{ml})$ of LPS was needed to induce a small but significant increase in the total number of platelet-neutrophil interactions in control human blood (Figure 5B). A comparison of platelet-neutrophil interactions in control or SCD blood before and after treatment with $0.25 \mu \mathrm{g} / \mathrm{ml}$ LPS (Figure $5 \mathrm{C}$ ) suggests that a concentration of LPS that is ineffective in control blood effectively promulgates neutrophil-platelet aggregate nucleation in SCD blood. Pretreatment of SCD human blood with LPS at concentrations greater than $0.25 \mu \mathrm{g} / \mathrm{ml}$ resulted in blood aggregation, thus rendering the blood unfit for qMFM studies. To establish that the increase in neutrophil-platelet interactions in SCD blood following a low dose of LPS was a result of TLR-4 activation, we conducted a separate set of qMFM studies in the presence of a TLR-4 inhibitor. Figure 5D demonstrates that treatment with a TLR-4 antagonist, TAK-242 $(50 \mu \mathrm{g} / \mathrm{ml})$ led to a significant reduction in the number of platelet-neutrophil interactions in LPS-pretreated SCD blood. Following treatment with TAK-242, the platelet-neutrophil interactions in LPS-treated SCD blood were attenuated to a level below that observed in control blood at baseline (without LPS treatment). The interactions were also slightly reduced following treatment with the vehicle intralipid used in the reconstitution of TAK-242 (Figure 5D); however, this reduction was small compared with TAK-242 treatment, suggesting that the reduction in interactions observed following TAK242 treatment was specific to TLR-4 inhibition (Figure 5D). TAK-242 had no significant effect on the number of arresting neutrophils (Figure 5E), suggesting that the TAK-242-induced reduction in platelet-neutrophil interactions was not a result of neutrophil detachment, but rather a consequence of the inability of platelets to adhere to neutrophils under vascular mimetic flow.

LPS-induced neutrophil-platelet aggregation is P-selectin and Mac-1 dependent. Next, we tested whether simultaneous inhibition of platelet P-selectin and neutrophil Mac-1 ameliorates LPS-triggered neutrophil-plate- 
let interactions in SCD human blood. Control and steady-state SCD human blood was preincubated with LPS at the respective potent concentrations of 1 and $0.25 \mu \mathrm{g} / \mathrm{ml}$, and the efficacy of simultaneous inhibition of platelet P-selectin and neutrophil Mac-1 was determined using the experimental approach described in Supplemental Methods. Anti-P-selectin/CD11b Abs significantly attenuated neutrophil-platelet aggregate nucleation in SCD blood, evidenced by the substantial reduction in total platelet-neutrophil interactions (Figure 6A) and platelet interactions per neutrophil (Figure 6B). As shown in Figure 6, A and B, the neutrophil-platelet interactions after treatment with anti-P-selectin/CD11b Abs were significantly lower and nearly identical in both $1 \mu \mathrm{g} / \mathrm{ml}$ LPS-treated control and $0.25 \mu \mathrm{g} / \mathrm{ml}$ LPS-treated SCD human blood. The attenuation in neutrophil-platelet interactions seemed to be a direct effect of the inability of platelets to stay attached to arrested neutrophils under flow, which was evidenced by the significant increase in percent interactions with lifetimes of less than 10 seconds (Supplemental Figure 12, A and B). Although the percent of interactions with lifetimes greater than 10 seconds also decreased after anti-P-selectin/CD11b Abs treatment, the difference did not reach significance due to the small number of events lasting more than 10 seconds. Also, anti-PSGL1 (CD162) and anti-GPIb $\alpha$ (CD42b) function-blocking Abs significantly reduced both the total platelet-neutrophil interactions and the number of platelet interactions per neutrophil (Supplemental Figure 13) in LPS-treated SCD human blood under flow. Treatment with a control isotype-matched $\mathrm{IgG}_{1} \mathrm{Ab}$ did not inhibit LPS-induced neutrophil-platelet interactions (Figure 6, C and D), suggesting that the reduction in interactions achieved after $\mathrm{Ab}$ treatment was specific to the inhibition of P-selectin-PSGL-1 and Mac-1-GPIb $\alpha$ binding between freely flowing platelets and arrested neutrophils.

$P$-selectin inhibition ameliorates pulmonary arteriolar microembolism in SCD mice. qFILM revealed that therapeutic inhibition of platelet P-selectin serves to prevent $0.1 \mu \mathrm{g} / \mathrm{kg}$ IV LPS-triggered pulmonary vaso-occlusion in SCD mice by disintegrating large neutrophil-platelet aggregates into smaller aggregates that are too small to trap in the pre-capillary arteriolar bottlenecks. Inspired by the in vitro qMFM findings in SCD human blood, we decided to use FFILM to test whether blockade of platelet P-selectin can lead to a reduction of neutrophil-platelet aggregates and an attenuation of $0.1 \mu \mathrm{g} / \mathrm{kg}$ IV LPS-induced pulmonary arteriole microembolism in SCD mice in vivo. Function-blocking anti-mouse P-selectin mAb Fab fragments (anti-PFab) were administered via tail vein along with $0.1 \mu \mathrm{g} / \mathrm{kg}$ IV LPS. Anti-PFab significantly attenuated LPS-induced occlusion of pulmonary arteriolar bottlenecks in SCD mice (Figure 7). A majority of FOVs observed with qFILM were free of pulmonary vaso-occlusions in SCD mice given $0.1 \mu \mathrm{g} / \mathrm{kg}$ IV LPS plus anti-PFab (Figure 7A and Supplemental Video 20). Blocking P-selectin reduced the frequency and extent of pulmonary vaso-occlusions to levels similar to those in SCD mice given IV saline (Figure 7 compared with Figure 1). Identical to the observations made in SCD mice administered IV saline (Supplemental Figure 2), only small neutrophil-platelet aggregates were occasionally observed to occlude the arteriolar bottlenecks in SCD mice administered $0.1 \mu \mathrm{g} / \mathrm{kg}$ IV LPS plus anti-PFab. A characteristic small neutrophil-platelet aggregate containing a few neutrophils and platelets can be seen occluding the pulmonary arteriolar bottleneck in Figure 7B. SCD mice administered $0.1 \mu \mathrm{g} / \mathrm{kg}$ IV LPS plus anti-PFab had a significantly reduced average number of pulmonary vaso-occlusions per FOV and a significantly smaller percent of FOVs with pulmonary vaso-occlusions compared with $0.1 \mu \mathrm{g} / \mathrm{kg}$ IV LPS SCD mice (Figure 7 , $\mathrm{C}$ and $\mathrm{D})$. The average number of pulmonary vaso-occlusions per FOV and percent FOVs with pulmonary vaso-occlusions in SCD mice administered $0.1 \mu \mathrm{g} / \mathrm{kg}$ IV LPS plus anti-PFab were comparable to those observed in SCD mice administered IV saline (0.52 pulmonary vaso-occlusions per FOV in Figure $1 \mathrm{C}$ and $38 \%$ FOV with pulmonary vaso-occlusions in Figure 1D). Anti-PFab administration led to a significant reduction in the average number of neutrophil vaso-occlusions (Figure 7E), which were shown in Figure 1I to be the predominant type of pulmonary vaso-occlusions occurring in SCD mice administered $0.1 \mu \mathrm{g} / \mathrm{kg}$ IV LPS. We hypothesized that P-selectin inhibition could enable a reduction in pulmonary vaso-occlusions through the disintegration of large neutrophil-platelet aggregates into smaller aggregates, which were small enough to transit through the arteriolar bottlenecks. Remarkably, Figure 7, F and G, and Supplemental Video 21 demonstrate that P-selectin inhibition enabled disintegration of neutrophil-platelet aggregates in the pulmonary arterioles of SCD mice in vivo. Figure $7 \mathrm{G}$ and Supplemental Video 21 show a neutrophil-platelet aggregate (comprising 3 neutrophils attached to a few platelets) as it arrives in the pulmonary arteriole, rapidly enters the arteriolar bottleneck, and then disintegrates, releasing a neutrophil that quickly enters into the capillaries by the end of the video. To validate this hypothesis, we analyzed the effect of anti-PFab administration on the size of pulmonary vaso-occlusions. Anti-PFab administration significantly reduced the number of large (area $>1,000 \mu \mathrm{m}^{2}$ ) pulmonary vaso-occlusions in SCD mice challenged with $0.1 \mu \mathrm{g} / \mathrm{kg}$ 
IV LPS plus anti-PFab (Figure 7H), suggesting that P-selectin inhibition contributes to a reduction in the size of neutrophil-platelet aggregates in SCD mice in vivo. P-selectin inhibition did not significantly affect the small number of pulmonary vaso-occlusions in control mice (Supplemental Figure 14).

\section{Discussion}

Epidemiological evidence suggests that systemic vaso-occlusive pain crisis in SCD is often an antecedent to acute chest syndrome, a type of acute lung injury (3). The current treatment for acute chest syndrome is primarily supportive, and the molecular mechanism remains largely unknown. In a recent autopsy study (27), pulmonary histopathology of acute chest syndrome patients revealed occlusion of the pulmonary vasculature with aggregates of platelets or erythrocytes, suggesting that the molecular events surrounding pulmonary vaso-occlusion contribute to lung injury. Our data suggest that systemic vaso-occlusive crisis also produces pulmonary vaso-occlusion by platelet-neutrophil aggregates that arrest blood flow in the pulmonary arterioles. We propose that without specific therapy, typically exchange transfusion and antibiotics, these pulmonary vaso-occlusions can progress to the acute chest syndrome in SCD.

The current support for pulmonary vaso-occlusion stems primarily from histological studies of lungs in SCD mice, which reveal the presence of blood cell aggregates in the lung micro-vessels following challenge with hypoxia-reoxygenation (28), systemic LPS (29), or TNF (30). This study represents what we believe to be the first use of intravital microscopy (qFILM) to establish a paradigm for pulmonary vaso-occlusion in the intact lung of live SCD mice. We confirmed that pulmonary vaso-occlusion is absent or minimal in SCD mice following challenge with IV saline. Quantitative analysis revealed that a low-nanogram dose of 0.1 $\mu \mathrm{g} / \mathrm{kg}$ IV LPS, which is innocuous to control mice, selectively triggers pulmonary vaso-occlusion in SCD mice. The average diameter of a pulmonary capillary segment is smaller than the diameter of a neutrophil (31). As a result, the junction between an arteriole and a pulmonary capillary network can possibly serve as a natural bottleneck to restrict the transit of cellular aggregates. Unlike the systemic microcirculation where vaso-occlusion never occurs in arterioles (1), qFILM systematically revealed that pulmonary vaso-occlusion occurs in the precapillary pulmonary arterioles and involves occlusion of the arteriolar bottlenecks by large neutrophil-platelet aggregates, which either form in situ or arrive preformed to the lung as microemboli. Indeed, recent intravital studies have shown that nucleation of platelets on adhered neutrophils during acute inflammation results in the formation of neutrophil-platelet aggregates in both the systemic $(9,10)$ and pulmonary microcirculation of WT mice $(9,14)$. Our finding that pulmonary vaso-occlusion occurs in precapillary pulmonary arterioles is distinct from the current paradigm for systemic vaso-occlusion, which suggests that postcapillary venules are the principal site of vaso-occlusion (1). These new observations of arteriolar pulmonary vaso-occlusion are supported by recent clinical studies that reveal selective occlusion of pulmonary artery branches and arterioles by aggregates of platelets in lung autopsies (27) and CT scans (32) of SCD patients diagnosed with lung injury.

Using a whole blood microfluidic in vitro approach (qMFM), we were able to demonstrate that neutrophil-platelet interactions under vascular mimetic flow conditions are also significantly higher in steady-state SCD than control human blood. We also show that similar to what we observed in SCD mice, neutrophil-platelet interactions were further augmented selectively in SCD but not control human blood following pretreatment with a small concentration of LPS $(0.25 \mu \mathrm{g} / \mathrm{ml})$. Remarkably, the augmented neutrophil-platelet interactions in SCD human blood were completely attenuated by blocking the interaction of platelet P-selectin and GPIb $\alpha$ with neutrophil PSGL-1 and Mac-1, respectively. In fact, the simultaneous inhibition of platelet P-selectin and neutrophil Mac-1 was potent enough to reduce the magnitude of platelet-neutrophil interactions in SCD human blood to that observed in control human blood. These findings are consistent with several flow cytometry-based studies that have confirmed the presence of circulating preformed neutrophil-platelet aggregates in the blood of steady-state SCD patients $(12,13,33)$. Finally, we show that blocking P-selectin led to attenuation of pulmonary vaso-occlusions in SCD mice, which was primarily driven by the disintegration of large neutrophil-platelet aggregates into smaller aggregates that appear to rapidly transit through the arteriolar bottlenecks. Collectively, our mice and human data suggest that the inflammatory milieu in SCD promotes the formation of large neutrophil-platelet aggregates in response to an otherwise innocuous inflammatory stimulus. These aggregates serve to enable pulmonary vaso-occlusion by occluding the arteriolar bottlenecks in the lung. The disintegration or inhibition of neutrophil-platelet aggregates by targeting P-selectin on platelets leads to resolution of pulmonary vaso-occlusions. 
Recent studies have identified a role for TLR4 activation in $\operatorname{SCD}(4,23,34)$. The mutation in the $\beta$-globin gene affects only hemoglobin in erythrocytes (3). However, intraerythrocytic polymerization of hemoglobin S results in hemolysis and altered rheology, releasing red cell- and tissue-derived danger-associated molecular pattern molecules (DAMPs) and inciting ischemia-reperfusion events, both of which have been proposed to "prime" innate immune signaling pathways (4). A number of erythrocyte-derived DAMPs have been characterized, including hemoglobin, heme, ADP, and uric acid (4). Several recent studies using humanized SCD mice and blood samples from SCD patients have established that cell-free hemoglobin and heme released into the circulation during hemolysis serve to activate neutrophils, platelets, and the endothelium by scavenging NO and activating the TLR4 pathway, respectively $(4,23,30,34,35)$. In a recent study (17), the translocation of bacterial TLR4 ligands from the gut into the blood circulation was shown to promote neutrophil activation in SCD mice, suggesting that the activation of neutrophils by chronic hemolysis primes these cells to activate further when exposed to traces of TLR4 agonists. In support of this hypothesis, our data demonstrate that IV administration of less than $3 \mathrm{ng}$ of a TLR4 ligand LPS, which is innocuous at this dose in control mice, leads to occlusion of lung arterioles in SCD mice by neutrophil-platelet aggregates. How hemolysis promotes hypersensitivity of neutrophils and platelets to TLR4 agonists is currently unknown and will be elucidated in future studies. We found that the nanogram dose of IV LPS triggers vaso-occlusion in SCD but not control mice (Figure 1), while a 10-fold-higher dose of IV LPS (Supplemental Figure 7) triggered vaso-occlusion in control mice as well. This observation is consistent with the clinical presentation in SCD patients. While SCD patients as well as normal healthy humans can develop acute lung injury and pneumonia with severe injury or infection with highly pathogenic bacteria, SCD patients are exquisitely susceptible to acute lung injury when exposed to less severe triggers $(5,18,22,36)$.

Although SCD affects millions of people worldwide (2), hydroxyurea (HU) is the only FDA-approved drug for the prophylactic treatment of SCD and prevents hemoglobin S polymerization by inducing fetal hemoglobin expression (1). Despite high efficacy, disease activity and mortality remain high in patients taking HU (1), with the life expectancy of SCD patients in the United States still estimated to be 20 years less than that of individuals without the disease (37). It is imperative to identify new treatments to prevent and better manage systemic vaso-occlusive pain crisis, to increase the quality and quantity of life for SCD patients (38). Based on our findings and the recent work of other investigators, targeting adhesion molecules on platelets and/or neutrophils to prevent or disintegrate neutrophil-platelet aggregates can be a promising therapy for SCD patients, particularly for the prevention of acute chest syndrome in high-risk patients presenting with systemic vaso-occlusive pain crisis. Interestingly, a pan-selectin inhibitor, rivipansel (GMI-1070), has been shown in phase II studies to reduce the time to resolution of systemic vaso-occlusive pain crisis and opioid administration in SCD patients (39). In a separate phase I study, intravenous immunoglobulin (IVIG) was shown to inhibit Mac-1 activation on neutrophils in SCD patient blood (40). Recently, an oral P-selectin inhibitor was also shown to improve microvascular blood flow in SCD patients (41). Also SelG1, a humanized anti-P-selectin monoclonal Ab, is currently in phase II studies to prevent systemic vaso-occlusive pain crisis in SCD patients (42).

Our findings support testing such blockers in clinical trials and also highlight several key aspects that need to be addressed in designing future therapies to prevent systemic vaso-occlusive pain crisis. First, targeting P-selectin and Mac-1 to prevent neutrophil-platelet aggregation can be effective in preventing pulmonary vaso-occlusion, suggesting that current agents in clinical trials may be appropriately targeting this pathogenesis. Second, a combination of P-selectin and Mac-1 blockers may be more effective than individual blockers in preventing systemic vaso-occlusive crisis in SCD patients. This also warrants the need for designing small molecule drugs that can simultaneously block both the P-selectin-PSGL-1 and GPIb $\alpha-$ Mac-1 interactions. Third, P-selectin and Mac-1 blockers could be most effective when given prophylactically rather than after vaso-occlusive crisis, or early after admission for vaso-occlusive crisis, perhaps in more severely affected patients with risk factors such as dropping hemoglobin and platelet levels $(18,22)$. Finally, global inhibition of P-selectin and/or Mac-1 could affect host defense, due to the inability of neutrophils to recruit to sites of inflammation. SCD patients experience leukocytosis even under a steady-state condition $(11,12,43)$, and therefore, interference with neutrophil recruitment can cause an elevation in circulating neutrophils. With these potential risks in mind, the targeted delivery of P-selectin or Mac-1 blockers as payloads in liposomes or nanocarriers designed to recruit specifically to the site of vaso-occlusion may represent an alternative approach for targeting vaso-occlusive crisis and pulmonary vaso-occlusion. 
The objective of the current study was to establish the significance and elucidate the cellular, molecular, and biophysical mechanism of pulmonary vaso-occlusion in SCD. Based on the clinical evidence, 10\%$20 \%$ of SCD patients hospitalized with systemic vaso-occlusive pain crisis develop acute chest syndrome during the course of their hospitalization (5). Our current study suggests that neutrophil-platelet aggregates, formed systemically and in situ, embolize into pulmonary vascular arteriolar bottlenecks to occlude blood flow. This process is TLR-4, platelet P-selectin, and GPIb $\alpha$ dependent, and can be prevented and reversed with directed receptor blockade. The epidemiology of acute chest syndrome following hospitalization with systemic vaso-occlusive pain crisis also offers a window for prevention, provided that targeted therapies to prevent pulmonary vaso-occlusion are identified. Future investigations will address whether inhibiting pulmonary vaso-occlusion formation is therapeutically beneficial in ameliorating acute chest syndrome.

\section{Methods}

Reagents. See Supplemental Methods for details on reagents.

Mice. Female ( $\sim 3$ months old) BERK SCD mice (Tg[Hu-miniLCR $\left.\alpha_{1}{ }^{\mathrm{G}} \gamma^{\mathrm{A}} \gamma \delta \beta^{\mathrm{S}}\right], \mathrm{Hb} \alpha_{\mathrm{m}}{ }^{-/-}, \mathrm{Hb} \beta_{\mathrm{m}}{ }^{-/-}$) and littermate BERK non-sickle (control) mice (Tg[Hu-miniLCR $\left.\alpha_{1}{ }^{\mathrm{G}} \gamma^{\mathrm{A}} \gamma \delta \beta^{\mathrm{S}}\right], \mathrm{Hb} \alpha_{\mathrm{m}}{ }^{-1-} \mathrm{Hb} \beta_{\mathrm{m}}{ }^{+-}$) were used for all experiments $(6,7)$. BERK SCD and BERK control mice have a mixed genetic background consisting of C57BL/6, 129Sv, FVB/N, DBA/2, and Black Swiss strains. BERK SCD and BERK control mice have been widely used as a model for SCD and control mice, respectively $(8,10,17,30)$. BERK SCD and control mice were bred in a custom colony maintained by the Vascular Medicine Institute at the Jackson Laboratory. For further details, see Supplemental Methods.

$q F I L M$. See Supplemental Methods for further details on the experimental setup of qFILM, which has also been described in detail elsewhere (16).

Experimental design of qFILM studies. Approximately 2-2.5 hours prior to qFILM, control or SCD mice were administered IV (tail vein) with saline, LPS, or LPS with Fab fragments of function-blocking mAb against P-selectin. Several cohorts of experiments were performed with the dose of IV LPS varying from $1 \mu \mathrm{g} / \mathrm{kg}$ to $0.1 \mu \mathrm{g} / \mathrm{kg}$. Anti-P-selectin Fab fragments were prepared in house using the function blocking $\mathrm{mAb}$ rat $\mathrm{IgG}_{1}$ against mouse P-selectin (clone RB40.34) and the Fab preparation kit. qFILM was also performed without the tail vein injections to verify the existence or absence of pulmonary vaso-occlusion at baseline in SCD or control mice. Mice were initially anesthetized with an intraperitoneal injection of $125 \mathrm{mg} / \mathrm{kg}$ ketamine $\mathrm{HCl}, 12.5 \mathrm{mg} / \mathrm{kg}$ xlyazine, and $0.04 \mathrm{mg} / \mathrm{kg}$ atropine sulfate. During qFILM, mice received maintenance anesthesia of $1 \%-1.5 \%$ isoflurane. Just prior to qFILM, IV fluorescent dyes and $\mathrm{mAbs}$ against blood cell lineage markers were injected through a carotid artery catheter to enable the visualization of the lung microcirculation and in vivo staining of neutrophils, platelets, or erythrocytes, respectively. IV fluorescent dyes included $38 \mu \mathrm{g} /$ mouse Evans blue or $75 \mu \mathrm{g} / \mathrm{mouse}$ FITC-dextran. Neutrophils were labeled in vivo with $12 \mu \mathrm{g} /$ mouse of AF546-conjugated Ly6-G mAb, platelets were labeled in vivo with $7 \mu \mathrm{g}$ /mouse of V450-conjugated CD49b mAb, and erythrocytes were labeled in vivo with $35 \mu \mathrm{g} /$ mouse of FITC-conjugated TER-119 mAb. Ly6-G, CD49b, and TER-119 mAbs have been used previously for in vivo visualization of neutrophils $(44,45)$, platelets $(46,47)$, and erythrocytes (16), respectively, by intravital fluorescence microscopy. See Supplemental Methods for details on experimental design.

qFILM image processing and analysis. See Supplemental Methods for details on FFILM image processing and analysis of pulmonary arteriolar vaso-occlusions.

Flow cytometry of whole lung. See Supplemental Methods for details on flow cytometry analysis of neutrophil-platelet aggregates in digested whole lung.

Human blood sample collection. Fresh blood samples were drawn from 24 steady-state SCD (23 SS and $1 \mathrm{~S} /$ $\beta^{0}$ thalassemia) and 8 healthy race-matched control (7AA and 1 AS) subjects at the Adult Sickle Cell Clinic of the University of Pittsburgh Medical Center (see Table 1 for clinical characterization of human subjects). Only nonsmokers who were not on chronic blood transfusion and hydroxyurea were included in this study. Blood was drawn via venipuncture using a $21 \mathrm{G}$ vacutainer push-button needle (BD Biosciences) into a 10-ml syringe containing 200 units of heparin (Henry Schein). The blood-filled syringe was placed on a blood mixer to avoid clotting and used within 2 hours following blood draw. Blood (150 $\mu$ l) was added to a Microvette 100 potassium EDTA tube (Sarstedt) and analyzed with the Hemavet HV950 (Drew Scientific) for the assessment of hematocrit, $\mathrm{Hb}$, and blood cell counts.

qMFM. qMFM has been recently introduced by our group (19). AF647-conjugated CD16 and FITC-conjugated CD49b mAbs were added to $500 \mu 1$ blood (3:500 CD16 mAb; 1:250 CD49b mAb) in 
a 1-ml Eppendorf tube for in situ staining of neutrophils and platelets, respectively (19). Blood was perfused through a polydimethylsiloxane (PDMS/silicone)-based microfluidic device consisting of 4 identical micro-channels (30 $\mu \mathrm{m}$ high and $500 \mu \mathrm{m}$ wide) at a physiological wall shear stress of $6 \mathrm{dyn} / \mathrm{cm}^{2}(25)$. A wall shear stress of $6 \mathrm{dyn} / \mathrm{cm}^{2}$ is within the physiological range of the shear stress observed in arterioles and venules in vivo (25). The sides and roof of each micro-channel were made of silicone, while the bottom was made of glass and coated with a cocktail of recombinant human P-selectin-Fc $(2 \mu \mathrm{g} / \mathrm{ml})$, ICAM-1-FC $(10 \mu \mathrm{g} / \mathrm{ml})$, and IL-8 $(10 \mu \mathrm{g} / \mathrm{ml})$. The preparation of the adhesive substrate and assembly of the microfluidic device have been described previously in detail $(19,48)$. Platelet-neutrophil interactions in blood-perfused microfluidic channels were visualized using a 2-stage imaging strategy that was recently introduced by our group (19). See Supplemental Methods for details on experimental setup.

Experimental design of qMFM studies. Control or SCD human blood was perfused through microfluidic micro-channels, and footprints of arresting and crawling neutrophils were visualized using qMFM step 1 (Figure 3, A and B, left) for 2 minutes. After 2 minutes, platelets nucleating on top of arrested neutrophils were visualized for another 2 minutes using qMFM step 2 (Figure 3, A and B, right). The transition from qMFM step 1 to step 2 is shown in Figure 3 and Supplemental Videos 17 and 18. See Supplemental Methods for details on experimental design of P-selectin/CD11b Ab blocking, CD42b/CD162 Ab blocking, LPS treatment, and TLR4 inhibition qMFM studies.

qMFM image processing and analysis. See Supplemental Methods for details on qMFM image processing and analysis of neutrophil-platelet interactions.

SEM. See Supplemental Methods for details on SEM.

SIM. See Supplemental Methods for details on SIM.

Statistics. The mean number of platelet-neutrophil interactions, mean number of arresting neutrophils, mean number of platelet-neutrophil interactions per neutrophil, mean number of pulmonary vaso-occlusions per FOV, area of pulmonary vaso-occlusions, and cellular composition of pulmonary vaso-occlusions were compared between groups using the 2-tailed unpaired Student's $t$ test along with the Bonferroni correction when appropriate for multiple comparisons. Distributions of the lifetime of platelet-neutrophil interactions under different conditions were compared using the nonparametric Kruskal-Wallis $H$ test. Percent platelet-neutrophil interactions with a lifetime of $0-10$ seconds or greater than 10 seconds and the percentage of FOVs with pulmonary vaso-occlusions were compared between different groups using 4-fold table analyses with Bonferroni $\chi^{2}$ statistics. In the dot plots, error bars are presented as mean \pm SEM. A $P$ value of less than 0.05 was used to determine the statistical significance.

Study approval. All animal experiments were approved by the Institutional Animal Care and Use Committee of the University of Pittsburgh. Approval for blood collection from SCD and healthy control subjects at the Adult Sickle Cell Clinic of University of Pittsburgh Medical Center was granted by the Institutional Review Board at the University of Pittsburgh. Written informed consent was obtained from human subjects after the nature and possible consequences of the studies were explained.

\section{Author contributions}

MFB performed and analyzed the qFILM and flow cytometry experiments with the assistance of RV; ET assisted with characterization of SCD mice and generating anti-P-selectin mAb Fab fragments. MAJ performed and analyzed the qMFM, SEM, and SIM experiments. GJK and JJ provided blood samples from human subjects. MTG was involved in experimental design and manuscript writing. PS was responsible for experimental design, manuscript writing, and project supervision. PS, MFB, and MAJ wrote the manuscript with consultation and contribution from all coauthors.

\section{Acknowledgments}

The authors would like to thank Ekaterina Koltsova at Fox Chase Cancer Center, Philadelphia, for suggestions regarding flow cytometry data analysis. This study was supported by NIH-NHLBI 1R01HL128297-01 (PS), American Heart Association 11SDG7340005 (PS), and Vascular Medicine Institute startup funds (PS). MFB was supported by NIH-NHLBI training grant T32HL110849 and NIH-NHLBI F32 NRSA 1F32HL131216-01. MAJ was supported by NIH-NHLBI training grant T32HL076124 and the NSF Graduate Research Fellowship Program. The Nikon multiphotonexcitation microscope was funded by NIH grant 1S10RR028478-01. The Nikon structured illumination microscope was funded by NIH 1S10OD010625-01A1. 
Address correspondence to: Prithu Sundd, BST E1255, 200 Lothrop Street, Pittsburgh, Pennsylvania 15261, USA. Phone: 412.648.9103; E-mail: prs51@pitt.edu.

1. Manwani D, Frenette PS. Vaso-occlusion in sickle cell disease: pathophysiology and novel targeted therapies. Blood. 2013;122(24):3892-3898.

2. GBD 2013 Mortality Causes of Death Collaborators. Global, regional, and national age-sex specific all-cause and cause-specific mortality for 240 causes of death, 1990-2013: a systematic analysis for the Global Burden of Disease Study 2013. Lancet. 2015;385(9963):117-171.

3. Rees DC, Williams TN, Gladwin MT. Sickle-cell disease. Lancet. 2010;376(9757):2018-2031.

4. Gladwin MT, Ofori-Acquah SF. Erythroid DAMPs drive inflammation in SCD. Blood. 2014;123(24):3689-3690.

5. Miller AC, Gladwin MT. Pulmonary complications of sickle cell disease. Am J Respir Crit Care Med. 2012;185(11):1154-1165.

6. Pászty C, et al. Transgenic knockout mice with exclusively human sickle hemoglobin and sickle cell disease. Science. 1997;278(5339):876-878.

7. Manci EA, Hillery CA, Bodian CA, Zhang ZG, Lutty GA, Coller BS. Pathology of Berkeley sickle cell mice: similarities and differences with human sickle cell disease. Blood. 2006;107(4):1651-1658.

8. Hidalgo A, Chang J, Jang JE, Peired AJ, Chiang EY, Frenette PS. Heterotypic interactions enabled by polarized neutrophil microdomains mediate thromboinflammatory injury. Nat Med. 2009;15(4):384-391.

9. Sreeramkumar V, et al. Neutrophils scan for activated platelets to initiate inflammation. Science. 2014;346(6214):1234-1238.

10. Li J, et al. Neutrophil AKT2 regulates heterotypic cell-cell interactions during vascular inflammation. J Clin Invest. 2014;124(4):1483-1496.

11. Curtis SA, Danda N, Etzion Z, Cohen HW, Billett HH. Elevated Steady State WBC and Platelet Counts Are Associated with Frequent Emergency Room Use in Adults with Sickle Cell Anemia. PLoS One. 2015;10(8):e0133116.

12. Dominical VM, et al. Prominent role of platelets in the formation of circulating neutrophil-red cell heterocellular aggregates in sickle cell anemia. Haematologica. 2014;99(11):e214-e217.

13. Polanowska-Grabowska R, et al. P-selectin-mediated platelet-neutrophil aggregate formation activates neutrophils in mouse and human sickle cell disease. Arterioscler Thromb Vasc Biol. 2010;30(12):2392-2399.

14. Ortiz-Muñoz G, Mallavia B, Bins A, Headley M, Krummel MF, Looney MR. Aspirin-triggered 15-epi-lipoxin A4 regulates neutrophil-platelet aggregation and attenuates acute lung injury in mice. Blood. 2014;124(17):2625-2634.

15. Looney MR, Thornton EE, Sen D, Lamm WJ, Glenny RW, Krummel MF. Stabilized imaging of immune surveillance in the mouse lung. Nat Methods. 2011;8(1):91-96.

16. Bennewitz MF, Watkins SC, Sundd P. Quantitative intravital two-photon excitation microscopy reveals absence of pulmonary vaso-occlusion in unchallenged sickle cell disease mice. Intravital. 2014;3(2):e29748.

17. Zhang D, et al. Neutrophil ageing is regulated by the microbiome. Nature. 2015;525(7570):528-532.

18. Gladwin MT, Vichinsky E. Pulmonary complications of sickle cell disease. N Engl J Med. 2008;359(21):2254-2265.

19. Jimenez MA, Tutuncuoglu E, Barge S, Novelli EM, Sundd P. Quantitative microfluidic fluorescence microscopy to study vaso-occlusion in sickle cell disease. Haematologica. 2015;100(10):e390-e393.

20. Vichinsky EP, Styles LA, Colangelo LH, Wright EC, Castro O, Nickerson B. Acute chest syndrome in sickle cell disease: clinical presentation and course. Cooperative Study of Sickle Cell Disease. Blood. 1997;89(5):1787-1792.

21. Nagler M, Wuillemin WA. Patient self-management of oral anticoagulation with vitamin $\mathrm{K}$ antagonists in everyday practice: comment on Jennings, et al British Journal of Haematology, 2014, 167:600-607. Br J Haematol. 2015;169(4):600-601.

22. Vichinsky EP, et al. Causes and outcomes of the acute chest syndrome in sickle cell disease. National Acute Chest Syndrome Study Group. N Engl J Med. 2000;342(25):1855-1865.

23. Belcher JD, et al. Heme triggers TLR4 signaling leading to endothelial cell activation and vaso-occlusion in murine sickle cell disease. Blood. 2014;123(3):377-390.

24. Andonegui G, et al. Mice that exclusively express TLR4 on endothelial cells can efficiently clear a lethal systemic Gram-negative bacterial infection. J Clin Invest. 2009;119(7):1921-1930.

25. Sundd P, Pospieszalska MK, Cheung LS, Konstantopoulos K, Ley K. Biomechanics of leukocyte rolling. Biorheology. 2011;48(1):1-35.

26. Koltsova EK, et al. Genetic deletion of platelet glycoprotein Ib alpha but not its extracellular domain protects from atherosclerosis. Thromb Haemost. 2014;112(6):1252-1263.

27. Anea $\mathrm{CB}$, et al. Pulmonary platelet thrombi and vascular pathology in acute chest syndrome in patients with sickle cell disease. Am J Hematol. 2016;91(2):173-178.

28. Wallace KL, Linden J. Adenosine A2A receptors induced on iNKT and NK cells reduce pulmonary inflammation and injury in mice with sickle cell disease. Blood. 2010;116(23):5010-5020.

29. Holtzclaw JD, Jack D, Aguayo SM, Eckman JR, Roman J, Hsu LL. Enhanced pulmonary and systemic response to endotoxin in transgenic sickle mice. Am J Respir Crit Care Med. 2004;169(6):687-695.

30. Chen G, Zhang D, Fuchs TA, Manwani D, Wagner DD, Frenette PS. Heme-induced neutrophil extracellular traps contribute to the pathogenesis of sickle cell disease. Blood. 2014;123(24):3818-3827.

31. Doerschuk CM. Mechanisms of leukocyte sequestration in inflamed lungs. Microcirculation. 2001;8(2):71-88.

32. Mekontso Dessap A, et al. Pulmonary artery thrombosis during acute chest syndrome in sickle cell disease. Am J Respir Crit Care Med. 2011;184(9):1022-1029.

33. Frelinger AL, et al. Platelet activation and inhibition in sickle cell disease (pains) study. Platelets. 2014;25(1):27-35.

34. Ghosh S, et al. Extracellular hemin crisis triggers acute chest syndrome in sickle mice. J Clin Invest. 2013;123(11):4809-4820.

35. Cardenes N, et al. Platelet bioenergetic screen in sickle cell patients reveals mitochondrial complex V inhibition, which contributes to platelet activation. Blood. 2014;123(18):2864-2872. 
36. Strouse JJ, et al. Severe pandemic H1N1 and seasonal influenza in children and young adults with sickle cell disease. Blood. 2010;116(18):3431-3434.

37. Elmariah $\mathrm{H}$, et al. Factors associated with survival in a contemporary adult sickle cell disease cohort. Am J Hematol. 2014;89(5):530-535.

38. Telen MJ. Beyond hydroxyurea: new and old drugs in the pipeline for sickle cell disease. Blood. 2016;127(7):810-819.

39. Telen MJ, et al. Randomized phase 2 study of GMI-1070 in SCD: reduction in time to resolution of vaso-occlusive events and decreased opioid use. Blood. 2015;125(17):2656-2664

40. Manwani D, et al. Single-dose intravenous gammaglobulin can stabilize neutrophil Mac-1 activation in sickle cell pain crisis. Am J Hematol. 2015;90(5):381-385.

41. Kutlar A, et al. A potent oral P-selectin blocking agent improves microcirculatory blood flow and a marker of endothelial cell injury in patients with sickle cell disease. Am J Hematol. 2012;87(5):536-539.

42. Mandarino D, Kawar Z, Alvarez R, Falconer D, Rollins SA, Rother RP. Placebo-controlled, double-blind, first-in-human, ascending single dose and multiple dose, healthy subject study of intravenous-administered SelG1, a humanized anti-P-selectin antibody in development for sickle cell disease. Blood. 2013;122(21):970.

43. Miller ST, et al. Prediction of adverse outcomes in children with sickle cell disease. N Engl J Med. 2000;342(2):83-89.

44. Yipp BG, et al. Infection-induced NETosis is a dynamic process involving neutrophil multitasking in vivo. Nat Med. 2012;18(9):1386-1393

45. Yipp BG, Kubes P. Antibodies against neutrophil LY6G do not inhibit leukocyte recruitment in mice in vivo. Blood. 2013;121(1):241-242.

46. Jenne CN, Wong CH, Petri B, Kubes P. The use of spinning-disk confocal microscopy for the intravital analysis of platelet dynamics in response to systemic and local inflammation. PLOS ONE. 2011;6(9):e25109.

47. Wong CH, Jenne CN, Petri B, Chrobok NL, Kubes P. Nucleation of platelets with blood-borne pathogens on Kupffer cells precedes other innate immunity and contributes to bacterial clearance. Nat Immunol. 2013;14(8):785-792.

48. Sundd P, Gutierrez E, Pospieszalska MK, Zhang H, Groisman A, Ley K. Quantitative dynamic footprinting microscopy reveals mechanisms of neutrophil rolling. Nat Methods. 2010;7(10):821-824 